\title{
Application of Hyphenated Techniques in Speciation Analysis of Arsenic, Antimony, and Thallium
}

\author{
Rajmund Michalski, Sebastian Szopa, Magdalena Jabłońska, and Aleksandra Lyko \\ Institute of Environmental Engineering, the Polish Academy of Sciences, 34 Skłodowskiej-Curie Street, 41 819 Zabrze, Poland
}

Correspondence should be addressed to Rajmund Michalski, michalski@ipis.zabrze.pl

Received 31 October 2011; Accepted 21 December 2011

Academic Editors: I. Garrard and A. Hirabayashi

Copyright ( $) 2012$ Rajmund Michalski et al. This is an open access article distributed under the Creative Commons Attribution License, which permits unrestricted use, distribution, and reproduction in any medium, provided the original work is properly cited.

\begin{abstract}
Due to the fact that metals and metalloids have a strong impact on the environment, the methods of their determination and speciation have received special attention in recent years. Arsenic, antimony, and thallium are important examples of such toxic elements. Their speciation is especially important in the environmental and biomedical fields because of their toxicity, bioavailability, and reactivity. Recently, speciation analytics has been playing a unique role in the studies of biogeochemical cycles of chemical compounds, determination of toxicity and ecotoxicity of selected elements, quality control of food products, control of medicines and pharmaceutical products, technological process control, research on the impact of technological installation on the environment, examination of occupational exposure, and clinical analysis. Conventional methods are usually labor intensive, time consuming, and susceptible to interferences. The hyphenated techniques, in which separation method is coupled with multidimensional detectors, have become useful alternatives. The main advantages of those techniques consist in extremely low detection and quantification limits, insignificant interference, influence as well as high precision and repeatability of the determinations. In view of their importance, the present work overviews and discusses different hyphenated techniques used for arsenic, antimony, and thallium species analysis, in different clinical, environmental and food matrices.
\end{abstract}

\section{Speciation Analytics and Hyphenated Techniques}

Speciation, as word borrowed from biology, is a term describing the existence of various chemical and physical forms of a particular element while speciation analytics denotes the determination of those forms [1]. The notion of speciation is used in chemistry to determine the occurrence of diverse forms of a given element (e.g., element at various oxidation states or bound with different ligands) in the analysed sample. The forms might differ in physical and chemical characteristics as well as in the influence they exert on living organisms. In the last several decades, speciation analytics has become one of the most central issues in analytical chemistry [2].

Even though its cost is significant, speciation analytics has been gaining greater importance when it comes to solving problems that concern not only the determination of total element contents but also taking into account various forms of occurrence. It plays an exceptional role in the examination of biochemical cycles of selected chemical compounds, determination of toxicity and ecotoxicity of selected elements, food and pharmaceutical product quality control, technological process control, as well as health risk assessment and clinical analytics [3]. Within the speciation analytics framework, determination of substances produced and emitted into the environment by humans and the analysis of natural compounds (formed as a result of biochemical transformations in living organisms or in the environment) can be differentiated. Environmental analytics deals with the former group while the latter one involves the biochemical and ecotoxic examinations.

It is also reasonable to differentiate between chemical and physical speciation. It is possible to distinguish between screening speciation (searching for and determining selected chemical forms) and distribution speciation (searching for and determining selected chemical individuals in specific elements of the examined sample) in chemical speciation. 
Another division within chemical speciation concerns group speciation (searching for and determining specific groups or classes of chemical forms) and individual speciation (searching for and determining all chemical individuals present in the sample) [4]. When it comes to liquid sample analyses, the most common technique is the one developed by Florence and Batley [5].

According to this method, a water or wastewater sample filtered through a $0.45 \mu \mathrm{m}$ filter is divided into a solid phase and mobile one, in which the determinations of total metal contents as well as the metal labile and bound forms are carried out.

The division suggested by Tessier et al. [6] is recommended in the research concerning the speciation of heavy metals in bottom sediments. They distinguished and defined five fractions, that is, exchangeable metals, carbonate-bound metals, iron and manganese oxides-bound metals, organic matter-bound metals, and other mineral-bound metals. Nonetheless, this method of speciation does not allow the differentiation between oxidation states of elements, which may be of great importance when considering their toxicity.

The analyte determination completes the procedure in which sampling is one of the most crucial stages. It is particularly important in speciation analytics as even the routine processes, such as dilution, $\mathrm{pH}$ changes caused by sample preservation, temperature, and pressure alternations, can bring about irreversible transformations in the primary analyte form [7]. Particular difficulties appear when the sample is collected under conditions considerably dissimilar to those in which it is later analyzed. It happens for instance when samples are collected from the lower strata of water bodies. Pressure drops trigger the emission of gaseous elements. For example, if it is $\mathrm{CO}_{2}$, then the increase of the sample $\mathrm{pH}$, acid-base balance changing, and increasing stability of complexes as well as precipitation of sparingly soluble sediments follow.

The lability and changeability of the sample is crucial when biological material is studied. Microbiological, enzymatic, photochemical, and other processes whose character is often unclear and unexpected can still occur in these samples after their collection [8]. Lowering the detection limits of analytes to extremely low concentration levels resulted in the fact that methods used so far did not always meet the necessary requirements [9]. For that reason, there has been a tendency for several years to combine various methods and techniques. These combinations are known as the hyphenated techniques. A suitable hyphenated technique should be selective towards determined analytes, sensitive within a wide range of concentrations, and ought to enable possibly best identification of the determined substances.

In speciation analytics, chromatographic methods are largely used for separation [10] whereas spectroscopic ones are employed for detection [11, 12], albeit the application of other methods is also possible [13]. The application of hyphenated techniques entails perfect understanding of analytical methodologies and detailed knowledge of the apparatus. These are expensive systems used in scientific research rather than in routine analyses.
The earliest hyphenated techniques were developed by coupling gas chromatography with various detectors. The following systems were elaborated: GC-AAS (Gas Chromatography-Atomic Absorption Spectrometry), GC-AES (Gas Chromatography-Emission Atomic Spectrometry), GC-MS (Gas Chromatography-Mass Spectrometry) or GC-ICP-MS-TOF (Gas Chromatography-Inductively Coupled Plasma-Mass Spectrometry-Time of Flight Mass Spectrometry).

Due to technological reasons, systems employing liquid chromatography methods for separation of analyzed substances, such as HPLC-ICP-MS (High Performance Liquid Chromatography-Inductively Coupled Plasma-Mass Spectrometry), appeared in the market slightly later. It is often thought that there exist elements (or their specific chemical forms) essential for the proper functioning of living organisms and elements (or their compounds) that interfere with metabolic processes. To paraphrase Paracelsus, a physician and chemist living in the 16th century, what ought to be discussed are significant or toxic concentrations or the amounts of elements and their diverse chemical forms.

The results of toxicological tests indicate that in many cases it is not the total content of a given element but the share of its particular forms that has a decisive influence on living organisms. Due to that fact, the knowledge of various elemental forms is more important than the information on its total content [14]. Elements occurring in ionic forms predominantly demonstrate biological activity and toxicity affecting living organisms. Ion chromatography is the most popular method serving to separate and determine organic and inorganic ionic substances [15]. It is applied in the hyphenated techniques and speciation analytics mainly for the determination of selected water disinfection by-products [16], as well as ions and metalloids [9].

Among the most popular hyphenated techniques used to determine different ionic forms of metals and metalloids, there are couplings of various liquid chromatography types. These include HPLC (High Performance Liquid Chromatography), IC (Ion Chromatography), I-EC (IonExclusion Chromatography), or SEC (Size Exclusion Chromatography) with ICP MS (Inductively Coupled Plasma Mass Spectrometry) or ESI MS (Electrospray Ionization Mass Spectrometry) [17]. The most popular hyphenated techniques utilizing ion chromatography are IC-ICP-MS (Ion Chromatography_-Inductively Coupled Plasma-Mass Spectrometry), IC-ICP-OES (Ion Chromatography-Inductively Coupled Plasma-Optical Emission Spectrometry), and IC-MS (Ion Chromatography-Mass Spectrometry) $[18,19]$.

The application of mass spectrometry detection allows not only to obtain the information on the qualitive and quantitative content of the sample but also to determine the structure and molar masses of the analytes. The main difficulties in using mass spectrometry detector coupled with chromatographic methods result from the fact that it is necessary to maintain very low pressure in the spectrometer while separated analyte ions leave the chromatographic column under comparatively high pressure. 
While it was relatively easy to couple a gas chromatograph with mass spectrometry detector, a large amount of the eluate in the case of liquid chromatography was a major obstacle in the introduction of HPLC-ESI-MS system into the laboratory practice [20]. Various ionization sources can be utilized in the HPLC-ESI-MS apparatus. These include ESI (Electrospray Ionization), APCI (Atmospheric Pressure Chemical Ionization), or APPI (Atmospheric Pressure Photochemical Ionization). The range of these applications depends on the polarity and mass of the analytes as well as the eluent flow rate. MS detection can be carried out in SIM (Selected Ion Monitoring) or SM (Scan Mode) modes. The former provides the information on the analyte mass while the latter offers data regarding mass spectra and mass distribution. The problems concerning identification in the case of large molecules relate mainly to the fact that there is a higher number of possibilities that the obtained spectra will have the same ratios of mass to charge.

It is estimated that approxmately $50 \%$ of all publications on the subject of speciation analytics involve only five elements, that is, arsenic, selenium, mercury, chromium, and tin.

Further $30 \%$ of papers are devoted to copper, zinc, lead, and iron [21]. The following literature review discusses arsenic, antimony, and thallium. The described elements have complex physical and chemical characteristics and are of great interest for both toxicologists and analytical chemists. Among them, arsenic and its compounds are the best known and described. Less information on antimony is available while thallium and it compounds are still the most mysterious and unfamiliar.

\section{Arsenic}

Arsenic is a metalloid from group 15 of the periodic table. Its most important oxidation states are of $-3,+3$, and +5 . Arsenic compounds were known in the Antiquity. Later, it gained great importance in medicine; it is even said to have been the basis of the modern pharmacology. People started using organoarsenic compounds at the turn of the nineteenth and twentieth centuries.

They turned out to be far less toxic for humans and animals than the inorganic ones. Nevertheless, nearly all arsenic medicines were withdrawn from the market in the second part of the previous century. Even though they were highly effective, they were also carcinogenic. Nowadays, arsenic compounds are used in medicine only very seldom. However, the interest in employing arsenic trioxide in the antineoplastic therapy has increased in recent years [72].

Arsenic has also been used in semiconductor production (as gallium arsenide), improving the quality of selected alloys, chemical warfare weapon industry, and wood conservation. It has also been added to glass as it provides a greenish glow. In addition, arsenic compounds have been employed in tanning and as pigments and pesticides [73]. Arsenic occurs in several hundred minerals, mainly in pyrite, and in lead and copper ores. It also appears in nature in a number of organic compounds such as arsenate (arsenic acid) $-\mathrm{H}_{3} \mathrm{AsO}_{4}$, arsenite (arsenious acid) $-\mathrm{HAsO}_{2}$, dimethylarsonic acid DMAA- $\left(\mathrm{CH}_{3}\right)_{2} \mathrm{AsO}(\mathrm{OH})$, cacodylic acid $\left(\mathrm{CH}_{3}\right)_{2} \mathrm{AsO}_{2} \mathrm{H}$, dimethylarsine DMA- $\left(\mathrm{CH}_{3}\right)_{2} \mathrm{AsH}$, methylarsine, trimethylarsine oxide TMAO- $\left(\mathrm{CH}_{3}\right)_{3} \mathrm{AsO}$, tetramethylarsonium salts, arsenobetaine $\mathrm{AsB}-\left(\mathrm{CH}_{3}\right)_{3}$ $\mathrm{As}^{+} \mathrm{CH}_{2} \mathrm{COOH}$ and arsenocholine AsC- $\left(\mathrm{CH}_{3}\right)_{3} \mathrm{As}^{+} \mathrm{CH}_{2}$ $\mathrm{CH}_{2} \mathrm{OH}$.

Anthropogenic sources of arsenic and its compounds comprise by-product emissions in mining and smelting of nonferrous metal ores and fossil fuels combustion (mainly brown and hard coals). Total world production of arsenic amounted to approximately 75,000 tonnes in 2005 . Around three quarters of this amount was used for wood preservation and one fifth in agriculture for herbicide manufacturing. The remaining amount was employed in nonferrous alloys and glass production. Whereas in highly industrialized countries applications of arsenic and its compounds have become more and more limited, they are still broadly used in developed countries, even though their toxic characteristics are widely known. It is estimated that $20 \%$ of population in Bangladesh consume water highly contaminated with arsenic and its compounds [36]. Arsenic is a very mobile element. For that reason, it occurs in all units of the environment. It easily migrates from the lithosphere into the hydrosphere, and its content in natural water is highly diversified and determined by the ground type and water pollution. Regulations that are in force in most European countries state that the total arsenic content in soil ought not to exceed $20 \mathrm{mg} / 1 \mathrm{~kg}$ of soil. Its admissible amount in drinking water amounts to $10 \mu \mathrm{g} / \mathrm{L}$, although its concentrations can exceed even several dozen $\mathrm{mg} / \mathrm{L}$ in surface and underground water depending on geological conditions.

In the atmosphere, arsenic occurs mainly in the forms of $\mathrm{AsO}_{3}$ and volatile organic compounds. Its mean concentration ranges between $1 \mathrm{ng} / \mathrm{m}^{3}$ in the countryside, $2 \mathrm{ng} / \mathrm{m}^{3}$ in the urban areas, and up to $50 \mathrm{ng} / \mathrm{m}^{3}$ in industrial regions.

Selected arsenic compounds determined with its speciation analytics encompass:

(i) inorganic ones such as arsenite (arsenious acid)$\mathrm{HAsO}_{2}$ - and arsenate (arsenic acid) $-\mathrm{H}_{3} \mathrm{AsO}_{4}$ among the inorganic compounds, $\mathrm{H}_{2} \mathrm{AsO}_{4}{ }^{-}(\mathrm{pH}=$ 2-7), $\mathrm{HAsO}_{4}{ }^{2-}(\mathrm{pH}>7)$, and $\mathrm{H}_{3} \mathrm{AsO}_{3}(\mathrm{pH}<9)$ are stable;

(ii) organic ones such as monomethylarsine MMA$\mathrm{CH}_{3} \mathrm{AsH}_{2}$, monomethylarsonic acid MMAA- $\mathrm{CH}_{3}$ $\mathrm{AsO}(\mathrm{OH})_{2}$, dimethylarsine $\mathrm{DMA}-\left(\mathrm{CH}_{3}\right)_{2} \mathrm{AsH}$, dimethylarsonic acid DMAA- $\left(\mathrm{CH}_{3}\right)_{2} \mathrm{AsO}(\mathrm{OH})$, arsenobetaine $\mathrm{AsB}-\left(\mathrm{CH}_{3}\right)_{3} \mathrm{As}^{+} \mathrm{CH}_{2} \mathrm{COOH}$, arsenocholine AsC- $\left(\mathrm{CH}_{3}\right)_{3} \mathrm{As}^{+} \mathrm{CH}_{2} \mathrm{CH}_{2} \mathrm{OH}$, trimethylarsine oxide $\mathrm{TMAO}-\left(\mathrm{CH}_{3}\right)_{3} \mathrm{AsO}$, tetramethylarsonium ion $\mathrm{Me}_{4} \mathrm{As}^{+}-\left(\mathrm{CH}_{3}\right)_{4} \mathrm{As}^{+}$, and arsenic-containing ribosides arsenosugars-various sugar structures [117].

All arsenic compounds, to a higher or lesser extent, possess carcinogenic and protoplasmatic (they destroy bacteria cell walls) characteristics. However, the toxicity of arsenic compounds depends on the form in which they are 
consumed and on their mobility. In general, it is assumed that their harmfulness decreases in the following order: arsines $>$ inorganic arsenites $>$ organic trivalent compounds (arsenoxides), inorganic arsenates $>$ organic pentavalent compounds $>$ arsonium compounds $>$ elemental arsenic. Arsenobetaine and arsenocholine are considered nontoxic. The symptoms of the chronic poisoning appear usually after a few years. They may include various forms of kidney, lung, liver, skin, or urinary bladder neoplasms. A prolonged skin contact with the arsenic dust can cause several types of skin cancer. What is interesting, long-term ingestion of low doses of arsenic compounds increases the body resistance against acute poisoning. In December 2010, there appeared information about the discovery of arsenophile GFAJ-1 bacteria that were able to sustain its growth in culture medium in which phosphorous had been replaced with arsenic (http://www.nasa.gov/topics/universe/features/astrobiology_ toxic_chemical.html).

It was suggested that arsenic might have been incorporated into biomolecules (e.g., DNA) which maintained its proper biological activity in this form. Nevertheless, this information was denied in March 2011. Arsenic speciation analytics makes use of the hyphenated techniques, such as HPLC-ESI-MS, IC-MS, or IC-ICP-MS [118-120], to separate and determine particular forms of the element. The literature data concerning arsenic speciation analytics with various hyphenated techniques is given in Table 1.

\section{Antimony}

Antimony, correspondingly to arsenic, belongs to group 15 of the periodic table. The physical and chemical qualities of both elements are similar. In the past, these two elements and their compounds were often determined together [134]. Its compounds were used as cosmetics in the Ancient Egypt. The alchemists used to believe that where there was antimony, there was gold as well. Antimony is a blue-white brittle metalloid. It behaves like a metal in most reaction. However, in some reactions, it demonstrates nonmetal characteristics. It has a rare quality-its solid form is less dense than its liquid one (similarly to water).

Antimony occurs in coal seams (especially brown coal ones), diesel fuel, and gasoline. Its concentration in coal seams can be as high as $30 \mathrm{mg} / \mathrm{kg}$ and it can reach $100 \mathrm{mg} / \mathrm{kg}$ in ashes. On the other hand, its concentration in petroleum ranges between 0.001 and $0.1 \mathrm{mg} / \mathrm{kg}$ [111]. Antimony is a constituent of many alloys. It is also employed to produce fire retardants. Its compounds are used in medicine, and also in matches manufacturing, rubber vulcanization, china and ammunition production, as well as biomedicine, in which they are used as antiprotozoal agents and for treating tropical diseases. Antimony may occur at four oxidation states, that is, $-3,+3,+4$, and +5 . It occurs mainly in $\mathrm{Sb}^{3+}$ and $\mathrm{Sb}^{5+}$ forms in the biological and geochemical environment. It is present in all units of the environment and its natural background in various environmental matrices is highly diversified [135]. Its content does not usually exceed $1 \mu \mathrm{g} / \mathrm{L}$ in clean water and $500 \mathrm{mg} / \mathrm{kg}$ in rocks. Beside its natural sources, antimony also occurs as anthropogenic pollution. More than 20,000 tonnes of this element are exploited in Japan every year, whereas, interestingly, only 100 tonnes of arsenic, which is far more toxic, are used.

Antimony speciation is especially important in environmental and clinical analytics, as it is a toxic element whose bioavailability and reactivity depend not only on the oxidation state but also on the character of its particular compounds. In general, inorganic antimony compounds are more toxic than the organic ones. $\mathrm{Sb}$ (III) compounds are ten times more toxic than $\mathrm{Sb}(\mathrm{V})$ ones. On the other hand, the toxicity of antimony compounds is approximately ten times lesser than arsenic ones but it depends on their oxidation states and structure. Antimony in the elemental form is more toxic than its salts [14].

The biological role of antimony has not been fully understood yet. IARC (The International Agency for Research on Cancer) has concluded that there is enough evidence obtained from animal testing to recognize $\mathrm{Sb}_{2} \mathrm{O}_{3}$ as a carcinogenic compound [136]. However, the U.S. Environmental Protection Agency [137] and German Research Community [138] categorize antimony as a main pollutant but do not indicate its carcinogenicity.

The first works relating to antimony speciation were published in the early 1980s [139]. The speciation analytics of antimony and its compounds can be divided into determination of $\mathrm{Sb}(\mathrm{III})$ and $\mathrm{Sb}(\mathrm{V})$ and organic antimony compounds. The speciation is usually performed with gas or liquid chromatography. Capillary electrophoresis methods are also used [140]. What is important in the application of gas chromatography in speciation analytics of antimony and its compounds is the fact that all of its organic compounds are gases at room temperature. Unfortunately, they are very often unstable. $\mathrm{Sb}$ (III) and $\mathrm{Sb}(\mathrm{V})$ compounds are reduced to $\mathrm{SbH}_{3}$, which at room temperature is a toxic gas with garlic odor.

The speciation is carried out at two stages. First, the part of the sample undergoes reduction in the presence of high $\mathrm{pH}$ (without concentration). In these conditions, only $\mathrm{Sb}$ (III) compounds are reduced. Then, the other part of the sample is reduced and the amount of $\mathrm{Sb}(\mathrm{V})$ is calculated on the basis of the difference.

Organic antimony compounds determined in the environment include, among others, monomethylated methylstibonic amid $\left[\mathrm{MeSbO}(\mathrm{OH})_{2}\right]$, dimethylated dimethylstibinic amid $\left[\mathrm{Me}_{2} \mathrm{SbOOH}\right]$, monomethylstibine $\left[\mathrm{MeSbH}_{2}\right]$, and dimethylstibine $\left[\mathrm{Me}_{2} \mathrm{SbH}\right]$ [2]. Unfortunately, they are not available in the standard form and thus are obtained for the analytical needs in laboratories. Methylated $\mathrm{Sb}(\mathrm{V})$ compounds are usually reduced to suitable $\mathrm{Sb}$ (III) compounds, that is, $\mathrm{MeSbO}(\mathrm{OH})_{2}$ to $\mathrm{MeSbH}_{2}, \mathrm{Me}_{2} \mathrm{SbOOH}$ to $\mathrm{Me}_{2} \mathrm{SbH}$, and $\mathrm{Me}_{3} \mathrm{SbX}_{2}$ to $\mathrm{Me}_{3} \mathrm{Sb}$.

The most significant limitation related to separation methods based on gas chromatography is the fact that only the compounds undergoing the reduction process can be determined. Another problem is that $\mathrm{Sb}$ (III) is unstable and it easily oxidizes to $\mathrm{Sb}(\mathrm{V})$, hence many research papers indicate relatively high $\mathrm{Sb}(\mathrm{V})$ content in the analyzed samples. 
TABLE 1: Selected examples of application of hyphenated techniques in arsenic speciation.

\begin{tabular}{|c|c|c|c|c|c|}
\hline Analytes & Analytical column & Mobile phase & $\begin{array}{c}\text { Method of separation } \\
\text { and detection }\end{array}$ & Matrix & Reference \\
\hline $\begin{array}{l}\mathrm{As}^{3+}, \mathrm{As}^{5+} \\
\mathrm{MMA}, \mathrm{DMA}\end{array}$ & $\begin{array}{l}\text { Hamilton PRP-X100 } \\
\text { Dionex AS7, AG7 }\end{array}$ & $\begin{array}{l}75 \mathrm{mM} \mathrm{Na}_{3} \mathrm{PO}_{4} \\
2.5-50 \mathrm{mM} \mathrm{HNO}_{3}\end{array}$ & HPLC-ICP-MS & $\begin{array}{l}\text { Surface water, } \\
\text { mining water, } \\
\text { underground } \\
\text { water }\end{array}$ & {$[22]$} \\
\hline $\begin{array}{l}\mathrm{As}^{3+}, \mathrm{As}^{5+} \\
\mathrm{MMA}, \mathrm{DMA} \\
\mathrm{AB}, \mathrm{AC}\end{array}$ & $\begin{array}{l}\text { Waters IC-Pak CM/D } \\
\text { Waters Guard-Pak CM/D }\end{array}$ & $\begin{array}{l}\mathrm{NaHCO}_{3} / \mathrm{Na}_{2} \mathrm{CO}_{3} \\
\mathrm{HNO}_{3}\end{array}$ & HPLC-ICP-MS & Water & {$[23]$} \\
\hline $\begin{array}{l}\mathrm{As}^{3+}, \mathrm{As}^{5+} \\
\mathrm{MMA}, \mathrm{DMA}\end{array}$ & Hamilton PRP-X100 & $\begin{array}{l}10-200 \mathrm{mM} \\
\mathrm{NH}_{4} \mathrm{H}_{2} \mathrm{PO}_{4}\end{array}$ & HPLC-ICP-DRC-MS & Sediments & {$[24]$} \\
\hline $\begin{array}{l}\mathrm{As}^{3+}, \mathrm{As}^{5+} \\
\mathrm{MMA}, \mathrm{DMA}\end{array}$ & Hamilton PRP-X100 & $30 \mathrm{mM} \mathrm{NH}_{4} \mathrm{H}_{2} \mathrm{PO}_{4}$ & HPLC-ICP-MS & Soils & {$[25]$} \\
\hline $\begin{array}{l}\mathrm{As}^{3+}, \mathrm{As}^{5+} \\
\mathrm{MMA}, \mathrm{DMA} \\
\mathrm{AB}\end{array}$ & Hamilton PRP-X100 & $20 \mathrm{mM} \mathrm{NH}_{4} \mathrm{H}_{2} \mathrm{PO}_{4}$ & HPLC-ICP-DRC-MS & $\begin{array}{l}\text { Polluted waters, } \\
\text { leach }\end{array}$ & {$[26]$} \\
\hline $\begin{array}{l}\mathrm{As}^{3+}, \mathrm{As}^{5+} \\
\mathrm{MMA}, \mathrm{DMA} \\
\mathrm{AB}\end{array}$ & Dionex AG-11, AS-11 & $\mathrm{NaOH}, \mathrm{HNO}_{3}$ & HPLC-ICP-MS & Urine & {$[27]$} \\
\hline $\begin{array}{l}\mathrm{As}^{3+}, \mathrm{As}^{5+} \\
\mathrm{MMA}, \mathrm{DMA} \\
\mathrm{AB}, \mathrm{AC}\end{array}$ & Hamilton PRP-X100 & $\begin{array}{l}0.3 \% \mathrm{HNO}_{3}, 10 \% \\
\text { methanol }\end{array}$ & $\begin{array}{l}\text { HPLC-ICP-MS, } \\
\text { HPLC-ESI MS }\end{array}$ & Fish sauce & {$[28]$} \\
\hline $\mathrm{As}^{3+}, \mathrm{As}^{5+}$ & Hamilton PRP X-100 & $\mathrm{Na}_{2} \mathrm{CO}_{3}$ & HPLC-ICP-MS & Surface water & {$[29]$} \\
\hline $\begin{array}{l}\mathrm{As}^{3+}, \mathrm{As}^{5+} \\
\mathrm{MMA}, \mathrm{DMA}\end{array}$ & Dionex AS7 & $\mathrm{HNO}_{3}$ & $\begin{array}{l}\text { HPLC- ICP-MS, } \\
\text { HPLC- INAA }\end{array}$ & $\begin{array}{l}\text { Waters, rice } \\
\text { extracts }\end{array}$ & {$[30]$} \\
\hline $\mathrm{As}^{3+}, \mathrm{As}^{5+}$ & Dionex AS9 & $\begin{array}{l}\mathrm{NaOH}, \mathrm{Na}_{2} \mathrm{CO}_{3} \\
\mathrm{NaHCO}_{3}\end{array}$ & HPLC-SF-ICP-MS & Soils & {$[31]$} \\
\hline $\mathrm{As}^{3+}, \mathrm{As}^{5+}$ & Wescan Anion-S C18 & EDTA & HPLC- ICP-MS & $\begin{array}{l}\text { River waters, } \\
\text { sludge }\end{array}$ & {$[32]$} \\
\hline $\mathrm{As}^{3+}, \mathrm{As}^{5+}$ & Biosil 125 SEC & $\mathrm{CH}_{3} \mathrm{COONH}_{4}$ & HPLC- ICP-MS & Fish tissues & {$[33]$} \\
\hline $\mathrm{As}^{3+}, \mathrm{As}^{5+}$ & Waters IC-Pak A HC & $\mathrm{NaOH}, \mathrm{KNO}_{3}$ & HPLC- ICP-MS & Water, sludge & {$[34]$} \\
\hline $\begin{array}{l}\mathrm{As}^{3+}, \mathrm{As}^{5+} \\
\mathrm{MMA}, \mathrm{DMA} \\
\mathrm{AB}, \mathrm{AC}\end{array}$ & $\begin{array}{l}\text { Develosil C30-UG-5, } \\
\text { Chemcosorb 7SAX }\end{array}$ & $\begin{array}{l}\text { sodium } \\
\text { butanesulfonate, } \\
\text { malonic acid, } \\
\text { tetramethylammonium } \\
\text { hydroxide, methanol, } \\
\text { ammonium tartrate }\end{array}$ & HPLC- ICP-MS & $\begin{array}{l}\text { Biological and } \\
\text { environmental } \\
\text { samples }\end{array}$ & {$[35]$} \\
\hline $\begin{array}{l}\mathrm{As}^{3+}, \mathrm{As}^{5+} \\
\mathrm{MMA}, \mathrm{DMA} \\
\mathrm{AB}\end{array}$ & Dionex AG7, AS7 & $\begin{array}{l}\text { nitric acid, } \\
\text { 1-benzene-2-disulfonic } \\
\text { acid dipotassium salt }\end{array}$ & HPLC-ICP-MS & Seafood & {$[36]$} \\
\hline $\begin{array}{l}\mathrm{As}^{3+}, \mathrm{As}^{5+} \\
\mathrm{MMA}, \mathrm{DMA}\end{array}$ & Hamilton PRP-X100 & $\begin{array}{l}\mathrm{NH}_{4} \mathrm{H}_{2} \mathrm{PO}_{4}, \\
\mathrm{NH}_{4} \mathrm{HPO}_{4} \\
\mathrm{CH}_{3} \mathrm{COONH}_{4}, \\
\mathrm{NaHCO}_{3}, \mathrm{NH}_{4} \mathrm{NO}_{3}\end{array}$ & HPLC-ICP-MS & $\begin{array}{l}\text { Soils, plant } \\
\text { tissues }\end{array}$ & {$[37]$} \\
\hline $\mathrm{As}^{3+}, \mathrm{As}^{5+}$ & Dionex AG12A/AS 12A & $\begin{array}{l}\text { disodium carbonate, } \\
\text { sodium hydroxide, } \\
\text { methanol }\end{array}$ & HPLC-ICP-MS & $\begin{array}{l}\text { Iron rich water } \\
\text { samples }\end{array}$ & {$[38]$} \\
\hline $\begin{array}{l}\mathrm{As}^{3+}, \mathrm{As}^{5+} \\
\mathrm{MMA}, \mathrm{DMA}\end{array}$ & Hamilton PRP X100 & $\mathrm{NH}_{4} \mathrm{NO}_{3}$ & HPLC-ICP-MS & Water & {$[39]$} \\
\hline $\begin{array}{l}\mathrm{As}^{3+}, \mathrm{As}^{5+} \\
\mathrm{MMA}, \mathrm{DMA}\end{array}$ & Hamilton PRP X100, & $\mathrm{NH}_{4} \mathrm{H}_{2} \mathrm{PO}_{4}$ & HPLC- DF-ICP-MS & Cucumber sap & {$[40]$} \\
\hline $\begin{array}{l}\mathrm{As}^{3+}, \mathrm{As}^{5+} \\
\mathrm{MMA}, \mathrm{DMA} \\
\mathrm{AB}\end{array}$ & Hamilton PRP X100 & $\begin{array}{l}\mathrm{NH}_{4} \mathrm{H}_{2} \mathrm{PO}_{4}, \\
\mathrm{NH}_{4} \mathrm{HPO}_{4}, \mathrm{MeOH}\end{array}$ & HPLC-ICP-MS & $\begin{array}{l}\text { Rice, soil, straw, } \\
\text { hair, nails }\end{array}$ & {$[41]$} \\
\hline $\begin{array}{l}\mathrm{As}^{3+}, \mathrm{As}^{5+} \\
\mathrm{MMA}, \mathrm{DMA} \\
\mathrm{AB}, \mathrm{AC} \\
\text { TMAO }\end{array}$ & $\begin{array}{l}\text { Hamilton PRP X100, } \\
\text { Zorbax 300-SCX }\end{array}$ & pyridine, $\mathrm{NH}_{4} \mathrm{H}_{2} \mathrm{PO}_{4}$ & HPLC-SF-MS & $\begin{array}{l}\text { Water, } \\
\text { sediments, } \\
\text { plants }\end{array}$ & {$[42]$} \\
\hline
\end{tabular}


Table 1: Continued.

\begin{tabular}{|c|c|c|c|c|c|}
\hline Analytes & Analytical column & Mobile phase & $\begin{array}{c}\text { Method of separation } \\
\text { and detection }\end{array}$ & Matrix & Reference \\
\hline $\begin{array}{l}\mathrm{As}^{3+}, \mathrm{As}^{5+} \\
\mathrm{DMA}\end{array}$ & G 3154A/101 & EDTA, $\mathrm{NH}_{4} \mathrm{H}_{2} \mathrm{PO}_{4}$ & HPLC-ICP-MS & Soils & {$[43]$} \\
\hline $\begin{array}{l}\mathrm{As}^{3+}, \mathrm{As}^{5+} \\
\mathrm{MMA}, \mathrm{DMA} \\
\mathrm{AB}\end{array}$ & 65001 and 65002 & $\mathrm{Na}_{2}$ EDTA, $\mathrm{NH}_{4} \mathrm{H}_{2} \mathrm{PO}_{4}$ & HPLC-ICP-MS & Urine & {$[44]$} \\
\hline $\begin{array}{l}\mathrm{As}^{3+} \\
\mathrm{As}^{5+}, \mathrm{MMA} \\
\mathrm{DMA}, \mathrm{AB}\end{array}$ & Supelcosil LC-SCX & $\begin{array}{l}\text { pyridine, } \mathrm{NaHCO}_{3}, \\
\mathrm{Na}_{2} \mathrm{CO}_{3}\end{array}$ & $\begin{array}{l}\text { HPLC-ICP-MS } \\
\text { HPLC-ES-MS }\end{array}$ & Hair, nail & {$[45]$} \\
\hline $\begin{array}{l}\mathrm{As}^{3+}, \mathrm{As}^{5+} \\
\mathrm{MMA}, \mathrm{DMA} \\
\mathrm{AB}, \mathrm{AC} \\
\text { TMAO }\end{array}$ & Dionex AG7, AS7 & $\mathrm{HNO}_{3}, \mathrm{MeOH}$ & HPLC-ICP-MS & Seafood & {$[46]$} \\
\hline $\begin{array}{l}\mathrm{As}^{3+}, \mathrm{As}^{5+} \\
\mathrm{MMA}, \mathrm{DMA} \\
\mathrm{AB}\end{array}$ & Dionex AS14, AS16, AS7 & $\mathrm{NaOH}, \mathrm{NH}_{4} \mathrm{H}_{2} \mathrm{PO}_{4}$ & HPLC-ICP-MS & Poultry wastes & {$[47]$} \\
\hline $\begin{array}{l}\mathrm{As}^{3+}, \mathrm{As}^{5+} \\
\mathrm{MMA}, \mathrm{DMA} \\
\mathrm{AB}\end{array}$ & $\begin{array}{l}\text { Shodex Asahipak } \\
\text { ES-502 N 7Ca }\end{array}$ & $\mathrm{HNO}_{3}, \mathrm{MeOH}$ & HPLC-ICP-MS & $\begin{array}{l}\text { Biological } \\
\text { samples }\end{array}$ & {$[48]$} \\
\hline $\begin{array}{l}\mathrm{As}^{3+}, \mathrm{As}^{5+} \\
\mathrm{MMA}, \mathrm{DMA} \\
\mathrm{AB}\end{array}$ & Hamilton PRP X100 & $\begin{array}{l}\left(\mathrm{NH}_{4}\right)_{2} \mathrm{SO}_{4} \\
\left(\mathrm{NH}_{4}\right)_{3} \mathrm{PO}_{4} \\
\mathrm{NH}_{4} \mathrm{HCO}_{3}\end{array}$ & HPLC-ICP-MS & Urine & {$[49]$} \\
\hline $\begin{array}{l}\mathrm{As}^{3+}, \mathrm{As}^{5+} \\
\mathrm{MMA}, \mathrm{DMA}\end{array}$ & Hamilton PRP X100 & $\mathrm{MeOH}, \mathrm{NH}_{4} \mathrm{H}_{2} \mathrm{PO}_{4}$ & HPLC-ICP-MS & Peanut butter & {$[50]$} \\
\hline $\begin{array}{l}\mathrm{As}^{3+}, \mathrm{As}^{5+} \\
\mathrm{MMA}, \mathrm{DMA}\end{array}$ & Hamilton PRP X100 & $\begin{array}{l}\left(\mathrm{NH}_{4}\right)_{2} \mathrm{HPO}_{4} \\
\mathrm{NH}_{4} \mathrm{H}_{2} \mathrm{PO}_{4}\end{array}$ & HPLC-ICP-MS & Wool & {$[51]$} \\
\hline $\begin{array}{l}\mathrm{As}^{3+}, \mathrm{As}^{5+} \\
\mathrm{MMA}, \mathrm{DMA} \\
\mathrm{AC}, \mathrm{AB} \\
\text { TMAO, } \\
\text { TMAs }\end{array}$ & Hamilton PRP X100 & $\begin{array}{l}\mathrm{NH}_{4} \mathrm{HPO}_{4}, \\
\left(\mathrm{NH}_{4}\right)_{2} \mathrm{HCO}_{3}, \\
\text { pyridine, } \mathrm{MeOH}\end{array}$ & $\begin{array}{l}\text { HPLC-ICP-MS, } \\
\text { HPLC-ES-MS }\end{array}$ & $\begin{array}{l}\text { Chinese } \\
\text { seaweeds }\end{array}$ & {$[52]$} \\
\hline $\begin{array}{l}\mathrm{As}^{3+}, \mathrm{As}^{5+} \\
\mathrm{MMA}, \mathrm{DMA} \\
\mathrm{AB}\end{array}$ & Hamilton PRP X100 & $\begin{array}{l}\mathrm{NH}_{4} \mathrm{HPO}_{4} \\
\mathrm{NH}_{4} \mathrm{H}_{2} \mathrm{PO}_{4}\end{array}$ & HPLC-ICP-MS & $\begin{array}{l}\text { Biological } \\
\text { samples (fish, } \\
\text { rice, chicken) }\end{array}$ & {$[53]$} \\
\hline $\begin{array}{l}\mathrm{As}^{3+}, \mathrm{As}^{5+} \\
\mathrm{MMA}, \mathrm{DMA} \\
\mathrm{AB}\end{array}$ & Dionex AS7 & $\mathrm{NH}_{4} \mathrm{H}_{2} \mathrm{PO}_{4}, \mathrm{NH}_{4} \mathrm{OH}$ & HPLC-ICP-MS & Waters & {$[54]$} \\
\hline $\begin{array}{l}\mathrm{As}^{3+}, \mathrm{As}^{5+} \\
\mathrm{MMA}, \mathrm{DMA} \\
\mathrm{AB}, \mathrm{AC} \\
\text { TMAO, } \\
\text { TMAI }\end{array}$ & Excelpak CHA-E11 & $\mathrm{HNO}_{3}$ & HPLC-ICP-MS & Rats urine & {$[55]$} \\
\hline $\begin{array}{l}\mathrm{As}^{3+}, \mathrm{As}^{5+} \\
\mathrm{MMA}, \mathrm{DMA} \\
\mathrm{AB}, \mathrm{AC}\end{array}$ & Dionex AS7, AG7 & $\mathrm{NaHCO}_{3}, \mathrm{Na}_{2} \mathrm{CO}_{3}$ & HPLC-ICP-MS & $\begin{array}{l}\text { Fish, mussel } \\
\text { extracts }\end{array}$ & {$[56]$} \\
\hline $\begin{array}{l}\mathrm{As}^{3+}, \mathrm{As}^{5+} \\
\mathrm{MMA}, \mathrm{DMA} \\
\mathrm{AB}, \mathrm{AC} \\
\mathrm{TMAO} \\
\mathrm{TeMAs}^{+}\end{array}$ & Dionex AS7, AG7 & $\begin{array}{l}\mathrm{HNO}_{3} \text {, } \\
\text { benzene-1,2-disulfonic } \\
\text { acid }\end{array}$ & HPLC-ICP-MS & Fish oil & {$[57]$} \\
\hline $\begin{array}{l}\mathrm{As}^{3+}, \mathrm{As}^{5+} \\
\mathrm{MMA}, \mathrm{DMA} \\
\mathrm{AC}, \mathrm{AB} \\
\text { TMAO, } \\
\mathrm{TeMAs}^{+}\end{array}$ & Dionex AG4, AS4A & $\mathrm{HNO}_{3}$ & HPLC-ICP-MS & Marine samples & {$[58]$} \\
\hline $\begin{array}{l}\mathrm{As}^{3+}, \mathrm{As}^{5+} \\
\mathrm{MMA}, \mathrm{DMA}\end{array}$ & Hamilton PRP X100 & $\left(\mathrm{NH}_{4}\right)_{2} \mathrm{HPO}_{4}, \mathrm{MeOH}$ & HPLC-ICP-MS & Soil extracts & {$[59]$} \\
\hline
\end{tabular}


TABle 1: Continued.

\begin{tabular}{|c|c|c|c|c|c|}
\hline Analytes & Analytical column & Mobile phase & $\begin{array}{l}\text { Method of separation } \\
\text { and detection }\end{array}$ & Matrix & Reference \\
\hline $\mathrm{As}^{3+}, \mathrm{As}^{5+}$ & Hamilton PRP X100 & $\begin{array}{l}\mathrm{CH}_{3} \mathrm{COOH}, \mathrm{NH}_{4} \mathrm{NO}_{3} \\
\text { EDTA }\end{array}$ & HPLC-ICP-MS & Drinking water & {$[60]$} \\
\hline $\mathrm{As}^{3+}, \mathrm{As}^{5+}$ & Dionex AS12 & $\mathrm{NaHCO}_{3}, \mathrm{Na}_{2} \mathrm{CO}_{3}$ & HPLC-HGAAS & Mine tailings & {$[61]$} \\
\hline $\begin{array}{l}\mathrm{As}^{3+}, \mathrm{As}^{5+} \\
\text { MMA, DMA }\end{array}$ & - & - & FIA-HGAAS & Seawater & {$[62]$} \\
\hline $\mathrm{As}^{3+}$, Total As & - & $\begin{array}{l}0.029 \mathrm{M} \mathrm{HNO}_{3} \\
0.024 \mathrm{M} \mathrm{HCl}\end{array}$ & FIA-HGAAS & Water samples & {$[63]$} \\
\hline $\mathrm{As}^{3+}, \mathrm{As}^{5+}$ & - & - & FI-EHG-AAS & $\begin{array}{l}\text { Synthetic } \\
\text { samples }\end{array}$ & {$[64]$} \\
\hline $\begin{array}{l}\mathrm{As}^{3+}, \mathrm{As}^{5+} \\
\mathrm{MMA}, \mathrm{DMA}\end{array}$ & Hamilton PRP-X100 & $\begin{array}{l}12 \mathrm{mM}\left(\mathrm{NH}_{4}\right)_{2} \mathrm{HPO}_{4} \\
7.5 \mathrm{mM}\left(\mathrm{NH}_{4}\right)_{2} \mathrm{SO}_{4} \\
10 \mathrm{mM}\left(\mathrm{NH}_{4}\right)_{2} \mathrm{CO}_{3}\end{array}$ & HPLC-ICP-AES & $\begin{array}{l}\text { Synthetic } \\
\text { samples }\end{array}$ & {$[65]$} \\
\hline $\begin{array}{l}\mathrm{As}^{3+}, \mathrm{As}^{5+} \\
\mathrm{MMA}, \mathrm{DMA} \\
\mathrm{AC}, \mathrm{AB}\end{array}$ & Hamilton PRP-X100 & $\begin{array}{l}\mathrm{NH}_{4} \mathrm{H}_{2} \mathrm{PO}_{4} \\
\left(\mathrm{NH}_{4}\right)_{2} \mathrm{HPO}_{4}\end{array}$ & HPLC-NAA & Water samples & {$[66]$} \\
\hline $\begin{array}{l}\mathrm{As}^{3+}, \mathrm{As}^{5+} \\
\mathrm{MMA}, \mathrm{DMA}\end{array}$ & $\begin{array}{l}\text { LiChrospher } 100 \mathrm{RP}-18 \mathrm{e} \\
\text { adsorbent }\end{array}$ & $\begin{array}{l}\text { tetrabutylammonium } \\
\text { hydrogen sulfate }\end{array}$ & HPLC-ETAAS & Water samples & {$[67]$} \\
\hline $\begin{array}{l}\mathrm{As}^{3+}, \mathrm{As}^{5+} \\
\mathrm{MMA}, \mathrm{DMA}\end{array}$ & Hamilton PRP X-100 & $\begin{array}{l}2,5 \mathrm{mM} \mathrm{NaH}_{2} \mathrm{PO}_{4} \\
2,5 \mathrm{mM} \mathrm{Na}_{2} \mathrm{HPO}_{4}\end{array}$ & HPLC-HGAAS & Natural waters & {$[68]$} \\
\hline $\begin{array}{l}\mathrm{As}^{3+}, \mathrm{As}^{5+} \\
\mathrm{MMA}, \mathrm{DMA}\end{array}$ & $\begin{array}{l}\text { Alltech All-guard } \\
\text { Adsorbosphere SAX } 5 \mu \mathrm{m}\end{array}$ & $10 \mathrm{mM} \mathrm{KH}_{2} \mathrm{PO}_{4}$ & HPLC-HG-AFS & $\begin{array}{l}\text { Particulate } \\
\text { matter }\end{array}$ & {$[69]$} \\
\hline $\begin{array}{l}\mathrm{As}^{3+}, \mathrm{As}^{5+} \\
\mathrm{MMA}, \mathrm{DMA}\end{array}$ & Dionex AS11, AG11 & $\begin{array}{l}10 \mathrm{mM}-100 \mathrm{mM} \\
\mathrm{NaOH}\end{array}$ & HPLC-HG-AFS & Polluted soil & {$[70]$} \\
\hline $\begin{array}{l}\mathrm{As}^{3+}, \mathrm{As}^{5+} \\
\mathrm{MMA}, \mathrm{DMA} \\
\mathrm{AC}, \mathrm{AB}\end{array}$ & Spherisorb ODS/ $\mathrm{NH}_{2}$ & $\begin{array}{l}5 \mathrm{mM} \mathrm{NaH}_{2} \mathrm{PO}_{4}, 5 \mathrm{mM} \\
\mathrm{Na}_{2} \mathrm{HPO}_{4},\end{array}$ & $\begin{array}{l}\text { HPLC-MO-HG-AAS, } \\
\text { HPLC-ICP-MS }\end{array}$ & Water, urine & {$[71]$} \\
\hline
\end{tabular}

Various forms of liquid chromatography are a possible alternative as they allow simultaneous determination of $\mathrm{Sb}(\mathrm{III})$ and $\mathrm{Sb}(\mathrm{V})$ compounds. Analytes such as $\mathrm{Sb}(\mathrm{III})$, $\mathrm{Sb}(\mathrm{V})$, or $\mathrm{Me}_{3} \mathrm{SbX}_{2}$, are anions themselves and can be determined as ions.

The most important problems with respect to speciation analytics of antimony and its compounds encompass the following.

(i) Standards of antimony compounds are unavailable and unstable. $\mathrm{Sb}(\mathrm{III})$ and $\mathrm{Sb}(\mathrm{V})$ standards are unstable. There are no certified reference materials for antimony compounds and suitable standards are difficult to obtain in chemical reactions. Very few inorganic and gaseous $\mathrm{Me}_{3} \mathrm{Sb}$ compounds are commercially available. Only trimethylated pentavalent antimony species $\left(\mathrm{Me}_{3} \mathrm{SbCl}_{2}, \mathrm{MeSbBr}_{2}, \mathrm{Me}_{3} \mathrm{SbO}\right.$, $\left.\mathrm{Me}_{3} \mathrm{Sb}(\mathrm{OH})_{2}\right)$ are produced with suitable purity and sufficient amount.

(ii) There appear problems with low and ultra-low analyte contents in the samples, especially those with complex matrices [2].

(iii) There exist problems related to unsuitable peak resolution and detection. Peak tailing, especially in the case of $\mathrm{Sb}$ (III) peak, appears while using liquid chromatography. Sb(III) forms divalent (and sometimes trivalent) ions in water solutions that strongly react with the resin. Besides, $\mathrm{Sb}(\mathrm{III}), \mathrm{Sb}(\mathrm{V})$ and $\mathrm{Me}_{3} \mathrm{SbX}_{2}$ are similar and difficult to separate.

Antimony and its compounds are most often determined in various types of water, such as drinking, mineral, and surface water. The information on the content of different speciation forms of antimony is not ample. It usually concerns specific water bodies and rivers or soil and plants growing in particular areas. Analyses concerning antimony and its compounds contents in biomedical samples attract great attention. Analysts find the matrix of such samples (urine, living tissues) challenging. They contain a lot of proteins, macromolecular substances, and enzymes that can bind with antimony compounds and cause its oxidation or reduction. The toxic activity mechanism of these compounds has not been fully understood yet and they are employed in treatment of various diseases, including the tropical ones. The literature data concerning antimony speciation analytics with various hyphenated techniques is given in Table 2.

\section{Thallium}

Thallium is a nonmetal from group 16 of the periodic table. It is a soft and silverish metal. It resembles lead and its surface quickly discolors due to oxidation processes when it is left in the air. It reacts with dilute strong inorganic acids (with the exception of hydrochloric acid) and displaces hydrogen out of them. 
TABLE 2: Selected examples of application of hyphenated techniques in antimony speciation.

\begin{tabular}{|c|c|c|c|c|c|}
\hline Analytes & Analytical column & Mobile phase & $\begin{array}{l}\text { Method of separation } \\
\text { and detection }\end{array}$ & Matrix & Reference \\
\hline $\mathrm{Sb}^{3+}, \mathrm{Sb}^{5+}$ & Hamilton PRP-X100 & $15 \mathrm{mM} \mathrm{HNO}_{3}$ & HPLC- ICP-MS & Plants & {$[74]$} \\
\hline $\mathrm{Sb}^{3+}, \mathrm{Sb}^{5+}$ & $\begin{array}{l}\text { Develosil C30-UG-5, } \\
\text { Chemcosorb 7SAX }\end{array}$ & $\begin{array}{l}\text { Malonic acid, sodium, } \\
\text { 1-butylosulfonian, } \\
\text { ammonium citrate, } \\
\text { methanol }\end{array}$ & HPLC- ICP-MS & $\begin{array}{l}\text { Biological and } \\
\text { environmental } \\
\text { samples }\end{array}$ & {$[35]$} \\
\hline $\begin{array}{l}\mathrm{Sb}^{3+}, \mathrm{Sb}^{5+} \\
\mathrm{TMSbCl}_{2}\end{array}$ & $\begin{array}{l}\text { Hamilton PRP-X100, } \\
\text { Dionex AS 14, AG } 14\end{array}$ & $20 \mathrm{mM}$ EDTA & HPLC- ICP-MS & Urine & {$[75,76]$} \\
\hline $\mathrm{Sb}^{3+}, \mathrm{Sb}^{5+}$ & Hamilton PRP-X100 & Phthalic acid, EDTA & HPLC- ICP-MS & Soil & {$[77,78]$} \\
\hline $\mathrm{Sb}^{3+}, \mathrm{Sb}^{5+}$ & Hamilton PRP-X100 & $\begin{array}{l}10 \mathrm{mM} \text { EDTA, } 1 \mathrm{mM} \\
\text { phthalic acid }\end{array}$ & HPLC- ICP-MS & $\begin{array}{l}\text { Airborne } \\
\text { particulate } \\
\text { matter }\end{array}$ & {$[79,80]$} \\
\hline $\begin{array}{l}\mathrm{Sb}^{3+}, \mathrm{Sb}^{5+} \\
\mathrm{TMSbCl}_{2} \\
\mathrm{TMS}(\mathrm{OH})_{2}\end{array}$ & $\begin{array}{l}\text { Hamilton PRP-X100, } \\
\text { Asahipak GS520HG }\end{array}$ & 10 mM TMAH & HPLC-ICP-MS & $\begin{array}{l}\text { Airborne } \\
\text { particulate } \\
\text { matter }\end{array}$ & {$[81,82]$} \\
\hline $\mathrm{Sb}^{3+}, \mathrm{Sb}^{5+}$ & Hamilton PRP-X100 & $\begin{array}{l}10 \mathrm{mM} \text { EDTA, } 1 \mathrm{mM} \\
\text { phthalic acid }\end{array}$ & HPLC-ICP-MS & Soils & {$[77,78]$} \\
\hline $\begin{array}{l}\mathrm{Sb}^{3+}, \mathrm{Sb}^{5+} \\
\mathrm{TMSbCl}_{2}\end{array}$ & Hamilton PRP X-100 & $\begin{array}{l}50 \mathrm{mM} \text { diammonium } \\
\text { tartrate, } 20 \mathrm{mM} \mathrm{KOH}\end{array}$ & $\begin{array}{l}\text { IC-HG-AFS, } \\
\text { HPLC-ICP-MS }\end{array}$ & Plant extracts & {$[83]$} \\
\hline $\mathrm{Sb}^{3+}, \mathrm{Sb}^{5+}$ & $\begin{array}{l}\text { Silica-based } \\
\text { solid-phase extraction } \\
\text { cartridges }\end{array}$ & $\begin{array}{l}\text { Ammonium } \\
\text { pyrrolidine } \\
\text { dithiocarbamate }\end{array}$ & SPE-ICP-MS & Water & {$[84]$} \\
\hline $\mathrm{Sb}^{3+}, \mathrm{Sb}^{5+}$ & Hamilton PRP X-100 & 20 mM EDTA & HPLC-ICP-MS & Soil & {$[85]$} \\
\hline $\mathrm{Sb}^{3+}, \mathrm{Sb}^{5+}$ & Hamilton PRP X-100 & $\begin{array}{l}10 \mathrm{mM} \text { EDTA, } 1 \mathrm{mM} \\
\text { phthalic acid }\end{array}$ & HPLC-ICP-MS & $\begin{array}{l}\text { Thirteen } \\
\text { fractions of } \\
\text { airborne } \\
\text { particulate } \\
\text { matter }\end{array}$ & {$[86]$} \\
\hline $\mathrm{Sb}^{3+}, \mathrm{Sb}^{5+}$ & $\begin{array}{l}\text { Dimercaptosuccinic } \\
\text { acid chemically } \\
\text { modifying } \\
\text { mesoporous titanium } \\
\text { dioxide microcolumn }\end{array}$ & Water & SPE-ICP-OES & Natural waters & {$[87]$} \\
\hline $\begin{array}{l}\mathrm{Sb}^{3+}, \mathrm{Sb}^{5+} \\
\mathrm{TMSbCl} \\
\mathrm{TMS}(\mathrm{OH})_{2}\end{array}$ & $\begin{array}{l}\text { Hamilton PRP-X100, } \\
\text { Hamilton PRPX-200, } \\
\text { Supelcosil LC-SCX, } \\
\text { Hamilton PRP1, } \\
\text { Phenomenex Intersil } \\
5 \text { ODS }\end{array}$ & $\begin{array}{l}\mathrm{KH}_{2} \mathrm{PO}_{4} / \mathrm{K}_{2} \mathrm{HPO}_{4} \\
0.5-5 \mathrm{mM}, \\
\mathrm{KHCO}_{3} / \mathrm{K}_{2} \mathrm{CO}_{3} \\
1-50 \mathrm{mM} \text {, pyridine, } \\
\text { 2,6 dicarboxylic acid } \\
\text { (PDCA): } 5-20 \mathrm{mM} \\
\mathrm{EDTA}, 5-50 \mathrm{mM} \\
\mathrm{HNO}_{3}, 1-4 \mathrm{mM}, \\
\text { ethylenediamine }\end{array}$ & $\begin{array}{l}\text { HPLC-ICP-MS, } \\
\text { HPLC-FAAS }\end{array}$ & $\begin{array}{l}\text { Environmental } \\
\text { samples }\end{array}$ & {$[88]$} \\
\hline $\mathrm{Sb}^{3+}, \mathrm{Sb}^{5+}$ & Dionex AS14 & $\begin{array}{l}2 \mathrm{mM} \mathrm{NH}_{4} \mathrm{HCO}_{3}, \\
2.2-45 \mathrm{mM} \text { tartaric } \\
\text { acid }\end{array}$ & HPLC-ICP-MS & Fish extracts & {$[89]$} \\
\hline $\mathrm{Sb}^{3+}, \mathrm{Sb}^{5+}$ & - & $\begin{array}{l}\text { Chloranilic acid } \\
\text { (2,5-dichloro-3,6- } \\
\text { dihy-droxy-1,4- } \\
\text { benzoquinone) }\end{array}$ & HMDE & Water & {$[90]$} \\
\hline $\begin{array}{l}\mathrm{Sb}^{3+}, \mathrm{Sb}^{5+} \\
\mathrm{MMSb}^{4+} \\
\mathrm{DMSb}^{3+} \\
\mathrm{TMSb}^{2+}\end{array}$ & $\begin{array}{l}\text { Teflon tube packed } \\
\text { with SE-30 on } \\
\text { Chromosorb W-HP }\end{array}$ & Liquid nitrogen & HG-SPE-ICP-MS & Surface waters & {$[91]$} \\
\hline $\mathrm{Sb}^{3+}, \mathrm{Sb}^{5+}$ & - & $\begin{array}{l}40 \mathrm{mM} \text { thioglycolic } \\
\text { acid }\end{array}$ & $\begin{array}{l}\text { SPE-ICP-OES, } \\
\text { SPE-ICP-MS }\end{array}$ & $\begin{array}{l}\text { Water, yeast } \\
\text { extract }\end{array}$ & {$[92]$} \\
\hline
\end{tabular}


Table 2: Continued.

\begin{tabular}{|c|c|c|c|c|c|}
\hline Analytes & Analytical column & Mobile phase & $\begin{array}{l}\text { Method of separation } \\
\text { and detection }\end{array}$ & Matrix & Reference \\
\hline $\mathrm{Sb}^{3+}, \mathrm{Sb}^{5+}$ & Hamilton PRP-X100 & $\begin{array}{l}20 \mathrm{mM} \text { EDTA, } \\
2 \mathrm{mM} \mathrm{KHP}\end{array}$ & HPLC-ICP-MS & Waters & {$[93]$} \\
\hline $\mathrm{Sb}^{3+}$, Total Sb & - & $\begin{array}{l}\text { l-cysteine, } \\
\text { hydrochloric acid }\end{array}$ & HG -AFS & Herbs & {$[94]$} \\
\hline $\mathrm{Sb}^{3+}, \mathrm{Sb}^{5+}$ & - & $\begin{array}{l}\mathrm{H}_{2} \mathrm{O}, 0.05 \mathrm{M} \text { EDTA, } \\
0.25 \mathrm{M} \mathrm{H}_{2} \mathrm{SO}_{4}\end{array}$ & HG -AFS & Soil & [95] \\
\hline $\mathrm{Sb}^{3+}, \mathrm{Sb}^{5+}$ & Hamilton PRP-X100 & $\begin{array}{l}10 \mathrm{mM} \text { EDTA, } 1 \mathrm{mM} \\
\text { phthalic acid }\end{array}$ & HPLC-ICP-MS & Moat water & {$[79,80]$} \\
\hline $\mathrm{Sb}^{3+}, \mathrm{Sb}^{5+}$ & Hamilton PRP-X100 & $\begin{array}{l}20 \mathrm{mM} \text { EDTA, } \\
2 \mathrm{mM} \text { KHP }\end{array}$ & HPLC-ICP-MS & $\begin{array}{l}\text { Citrus juices } \\
\text { Mineral water }\end{array}$ & {$[96]$} \\
\hline $\mathrm{Sb}^{3+}, \mathrm{Sb}^{5+}$ & Hamilton PRP-X100 & $5 \mathrm{mM} \mathrm{HNO}_{3}$ & HPLC-ICP-MS & Cell extracts & {$[74]$} \\
\hline $\begin{array}{l}\mathrm{Sb}^{3+}, \mathrm{Sb}^{5+} \\
\mathrm{TMSbCl}_{2}\end{array}$ & Hamilton PRP-X100 & $\begin{array}{l}20 \mathrm{mM} \text { EDTA, } \\
2 \mathrm{mM} \mathrm{KHP}\end{array}$ & HPLC-HG-AFS & Sea water, & {$[97]$} \\
\hline $\mathrm{Sb}^{3+}, \mathrm{Sb}^{5+}$ & Hamilton PRP-X100 & EDTA $2-20 \mathrm{mM}$ & $\begin{array}{l}\text { HPLC-ICP-MS, } \\
\text { FI-HG-ICP-MS }\end{array}$ & $\begin{array}{l}\text { Biological } \\
\text { samples }\end{array}$ & {$[98]$} \\
\hline $\begin{array}{l}\mathrm{Sb}^{3+}, \mathrm{Sb}^{5+} \\
\mathrm{TMSbCl}_{2}\end{array}$ & Dionex AS15/AG15 & $\begin{array}{l}20 \mathrm{mM} \text { EDTA, } \\
\mathrm{NH}_{4} \mathrm{OH}, 1 \mathrm{mM} \text { EDTA }\end{array}$ & HPLC-ICP-MS & $\begin{array}{l}\text { Pteris vittata } \\
\text { samples }\end{array}$ & \\
\hline $\mathrm{Sb}^{3+}, \mathrm{Sb}^{5+}$ & $\begin{array}{l}\text { Microcolumn with } \\
\text { immobilized } \\
\text { aminoacid }\end{array}$ & $\mathrm{HCl}$ & HG-ICP-OES & Urine & [99] \\
\hline $\mathrm{Sb}^{3+}, \mathrm{Sb}^{5+}$ & Hamilton PRP-X100 & $\begin{array}{l}200 \mathrm{mM} \\
\text { diammonium tartrate }\end{array}$ & HPLC-HG-AFS & $\begin{array}{l}\text { Airborne } \\
\text { particulate } \\
\text { matter }\end{array}$ & {$[100]$} \\
\hline $\begin{array}{l}\mathrm{Sb}^{3+}, \mathrm{Sb}^{5+} \\
\mathrm{TMSbCl}_{2}\end{array}$ & Hamilton PRP-X100 & $\begin{array}{l}20 \mathrm{mM} \text { EDTA }+ \\
2 \mathrm{mM} \text { potassium } \\
\text { hydrogen phthalate, } \\
50 \mathrm{mM} \text { diammonium } \\
\text { hydrogen phosphate }\end{array}$ & HPLC-(UV)-HG-AFS & $\begin{array}{l}\text { Algae and } \\
\text { mollusk extracts }\end{array}$ & {$[101]$} \\
\hline $\begin{array}{l}\mathrm{Sb}^{3+}, \mathrm{Sb}^{5+} \\
\mathrm{TMSbO}\end{array}$ & Hamilton PRP-X100 & $\begin{array}{l}2 \mathrm{mM} \text { phthalic acid } \\
2 \mathrm{mM} \\
\text { 4-hydroxybenzoic } \\
\text { acid }\end{array}$ & $\begin{array}{l}\text { HPLC-ICP-MS, } \\
\text { HPLC-ICP-OES }\end{array}$ & $\begin{array}{l}\text { Surface water, } \\
\text { soil extracts }\end{array}$ & {$[102]$} \\
\hline $\begin{array}{l}\mathrm{Sb}^{3+}, \mathrm{Sb}^{5+} \\
\mathrm{TMSbCl}_{2}\end{array}$ & Hamilton PRP-X100 & $\begin{array}{l}\text { EDTA- } \\
\mathrm{K}_{2} \mathrm{HPO}_{4} 20 \mathrm{mM}\end{array}$ & HPLC-HG-AFS & Sediments & {$[103]$} \\
\hline $\begin{array}{l}\mathrm{Sb}^{3+}, \mathrm{Sb}^{5+} \\
\mathrm{TMSBCl}_{2}\end{array}$ & Hamilton PRP-X100 & $\begin{array}{l}20 \mathrm{mM} \text { EDTA, } 8 \mathrm{mM} \\
\text { KHP } 1 \mathrm{mM} \mathrm{K} \mathrm{K}_{2} \mathrm{CO}_{3}\end{array}$ & HPLC-HG-AFS & $\begin{array}{l}\text { Environmental } \\
\text { samples }\end{array}$ & {$[104]$} \\
\hline $\mathrm{Sb}^{3+}, \mathrm{Sb}^{5+}$ & Hamilton PRP-X100 & $\begin{array}{l}250 \mathrm{mM} \\
\text { diammonium } \\
\text { tartrate, } 20 \mathrm{mM} \mathrm{KOH}\end{array}$ & HPLC-ICP-MS & Volcanic ash & {$[105]$} \\
\hline $\mathrm{Sb}^{3+}, \mathrm{Sb}^{5+}$ & - & $\begin{array}{l}1.5 \mathrm{M} \mathrm{HAc}, 0.5 \mathrm{M} \\
\mathrm{H}_{2} \mathrm{SO}_{4}\end{array}$ & FIA-HG-AAS & $\begin{array}{l}\text { Biological } \\
\text { samples }\end{array}$ & {$[106]$} \\
\hline $\begin{array}{l}\mathrm{Sb}^{3+}, \mathrm{Sb}^{5+} \\
\mathrm{TMSbCl}_{2}\end{array}$ & $\begin{array}{l}\text { CETAC ION-120 } \\
\text { Dionex AS } 14\end{array}$ & $\begin{array}{l}2 \mathrm{mM} \mathrm{NH}_{4} \mathrm{HCO}_{3}, \\
1 \mathrm{mM} \text { tartaric acid, } \\
1.25 \mathrm{mM} \mathrm{EDTA}\end{array}$ & HPLC-ICP-MS & Tap water & {$[75,76]$} \\
\hline $\mathrm{Sb}^{3+}, \mathrm{Sb}^{5+}$ & - & $\begin{array}{l}5.0 * 10^{-5} \mathrm{M} \text { BPHA, } \\
\text { Triton X-114 }(0.20 \% \\
(\mathrm{v}=\mathrm{v}))\end{array}$ & SPE-FAAS & Water & {$[107]$} \\
\hline $\begin{array}{l}\mathrm{Sb}^{3+}, \mathrm{Sb}^{5+} \\
\mathrm{TMSbCl}_{2}\end{array}$ & Hamilton PRP-X100 & $\begin{array}{l}20 \mathrm{mM} \text { EDTA, } 2 \mathrm{mM} \\
\mathrm{KHP}, 50 \mathrm{mM} \\
\left(\mathrm{NH}_{4}\right)_{2} \mathrm{HPO}_{4}\end{array}$ & HPLC-HG-AFS & Sea water & {$[97]$} \\
\hline $\mathrm{Sb}^{3+}$, Total Sb & - & - & HS-SDME-ETAAS & Lake water, soils & {$[108]$} \\
\hline
\end{tabular}


TABle 2: Continued.

\begin{tabular}{|c|c|c|c|c|c|}
\hline Analytes & Analytical column & Mobile phase & $\begin{array}{l}\text { Method of separation } \\
\text { and detection }\end{array}$ & Matrix & Reference \\
\hline $\begin{array}{l}\mathrm{Sb}^{3+}, \mathrm{Sb}^{5+} \\
\mathrm{TMSbCl}_{2}\end{array}$ & $\begin{array}{l}\text { Hamilton PRP-X100, } \\
\text { Dionex AS4A-SC }\end{array}$ & $\begin{array}{l}12 \mathrm{mM} \text { tetra- } \\
\text { methylammonium } \\
\text { hydroxide } 3 \mathrm{mM} \\
\text { tetra- } \\
\text { methylammonium } \\
\text { hydroxide }\end{array}$ & HPLC-ICP-MS & $\begin{array}{l}\text { Environmental } \\
\text { samples }\end{array}$ & [109] \\
\hline $\mathrm{Sb}^{3+}, \mathrm{Sb}^{5+}$ & Dionex AS 14, AG 14 & $\begin{array}{l}2 \mathrm{mM} \text { ammonium } \\
\text { hydrogen carbonate, } \\
2.2 \mathrm{mM} \text { tartaric acid, } \\
2 \mathrm{mM} \text { ammonium } \\
\text { hydrogen carbonate, } \\
45 \mathrm{mM} \text { tartaric acid, }\end{array}$ & HPLC-ICP-MS & $\begin{array}{l}\text { Synthetic } \\
\text { samples }\end{array}$ & {$[110]$} \\
\hline $\mathrm{Sb}^{3+}, \mathrm{Sb}^{5+}$ & Hamilton PRP-X100 & $\begin{array}{l}250 \mathrm{mM} \\
\text { diammonium } \\
\text { tartrate, } 20 \mathrm{mM} \mathrm{KOH}\end{array}$ & $\begin{array}{l}\text { HPLC-HG-AFS, } \\
\text { HPLC-ICP-MS }\end{array}$ & Coal fly ash & {$[111]$} \\
\hline $\mathrm{Sb}^{3+}, \mathrm{Sb}^{5+}$ & Synchropak Q300 & $\begin{array}{l}5 \mathrm{mM} \text { EDTA, } 2 \mathrm{mM} \\
\text { phthalic acid }\end{array}$ & HPLC-ICP-MS & Tap water & {$[81,82]$} \\
\hline $\mathrm{Sb}^{3+}, \mathrm{Sb}^{5+}$ & Hamilton PRP-X100 & $\begin{array}{l}20 \mathrm{mM} \text { EDTA, } 2 \mathrm{mM} \\
\text { phthalic acid }\end{array}$ & $\begin{array}{l}\text { HPLC-ICP-MS, } \\
\text { HPLC-ESI-MS }\end{array}$ & $\begin{array}{l}\text { Yoghurt, juice, } \\
\text { urine }\end{array}$ & {$[112]$} \\
\hline $\begin{array}{l}\mathrm{Sb}^{3+}, \mathrm{Sb}^{5+} \\
\mathrm{TMSbCl}_{2}\end{array}$ & Phenomenex SAX-SB & $\begin{array}{l}100 \mathrm{mM} \text { ammonium } \\
\text { tartrate }\end{array}$ & HPLC-ICP-MS & $\begin{array}{l}\text { Synthetic } \\
\text { solutions }\end{array}$ & {$[113]$} \\
\hline $\begin{array}{l}\mathrm{Sb}^{3+}, \mathrm{Sb}^{5+} \\
\mathrm{TMSbCl}_{2}\end{array}$ & $\begin{array}{l}\text { ION-120, Supelcosil } \\
\text { SAX Hamilton } \\
\text { PRP-X100, Dionex AS } \\
\text { 14, Dionex AS } 9\end{array}$ & $\begin{array}{l}\mathrm{NH}_{4} \mathrm{HCO}_{3}, \mathrm{EDTA}, \\
\text { Tartaric acid }\end{array}$ & HPLC-HG-AAS & $\begin{array}{l}\text { Environmental } \\
\text { samples }\end{array}$ & {$[114]$} \\
\hline $\mathrm{Sb}^{3+}, \mathrm{Sb}^{5+}$ & Supelcosil LC-SAX 1 & $\begin{array}{l}50 \mathrm{mM} \text { ammonium } \\
\text { tetrate }\end{array}$ & HPLC-HG-AAS & Water & {$[115]$} \\
\hline $\mathrm{Sb}^{3+}, \mathrm{Sb}^{5+}$ & - & $3 \mathrm{~g} / \mathrm{L}$ L-cysteine & HG-ICP-AES & $\begin{array}{l}\text { Spiked water } \\
\text { samples }\end{array}$ & {$[116]$} \\
\hline
\end{tabular}

Thallium occurs at oxidation states of +1 and +3 in compounds. $\mathrm{Tl}^{+}$cations are colorless and thallium(I) hydroxide is a soluble strong base. $\mathrm{Tl}^{3+}$ ions can exist in a solution only if its $\mathrm{pH}$ is close to 0 . When it is higher, $\mathrm{Tl}(\mathrm{OH})_{3}$ precipitates [141]. Thallium compounds are highly toxic. The element is also toxic in the dust form as it oxidizes in the contact with air. Food and respiratory thallium poisonings are possible. One of the characteristic poisoning symptoms is hair loss preceded by hair follicle atrophy. Other signs include digestion disorders, pain, neuropsychiatric complications, as well as cardiovascular system damages [2]. In the past, thallium salts were often added to rodenticides.

The anthrophogenic environmental occurrence of thallium is caused by ore smelting, handling, and processing of waste products from the metallurgic industry [142]. Also, vegetation damages have been observed and researched around a cement plant using thallium-containing pyrite smelting residues [143]. Thallium occurs in the Earth crust and its mean amount equals to $0.6 \mathrm{mg} / \mathrm{kg}$. It is also found, as a trace element, in ore seams containing sulfur and potassium compounds. Thallium(I) salts are easily absorbed through the skin and they usually enter living organisms in this way. Food is one of the important sources of thallium and its compounds. Consequently, food control monitoring is essential. Therefore, it is very important to determine sources of thallium in food and to investigate dietary intakes of this element. Thallium is widely distributed in the vegetable kingdom and is rapidly taken up by the plant root. Thus, the thallium-transfer from soils to plants should be investigated with respect to the correlation between the chemical form and the plant uptake of this element.

Thallium and its compounds are determined in urine, saliva, tissue, and blood samples in clinical analyses [144]. Thallium is a cumulative poison of high toxicity and is without taste, smell, and other warning attributes. Thallium and its compounds can enter the organism of persons working with them through the respiratory organs, through the gastrointestinal tract and through the skin. Industrial thallium poisoning can either be acute or chronic, but in all cases it is characterized by a long duration and severity of its course [142].

All results demonstrate the importance and the needs for more thallium speciation studies in different matrices, in order to obtain fundamental data for the treatment of subjects who suffer from chronic or acute thallium intoxication.

The following analytical techniques are used in the thallium analytics: atomic absorption spectrometry, coulometry, 
TABLE 3: Selected examples of application of hyphenated techniques in thalium speciation.

\begin{tabular}{|c|c|c|c|c|c|}
\hline Analytes & Analytical column & Mobile phase & $\begin{array}{l}\text { Method of separation } \\
\text { and detection }\end{array}$ & Matrix & Reference \\
\hline $\mathrm{Tl}^{1+}, \mathrm{Tl}^{3+}$ & Chelex-100 resin column & $14 \% \mathrm{HNO}_{3}$ & $\begin{array}{l}\text { SPE-ICP-MS, } \\
\text { SPE-GFAAS }\end{array}$ & River waters & {$[121]$} \\
\hline $\mathrm{Tl}^{1+}, \mathrm{Tl}^{3+}$ & L-Tyr-CNTs & $10 \% \mathrm{HNO}_{3}$ & SPE-STPF-ETAAS & Tap waters & {$[122]$} \\
\hline $\mathrm{Tl}^{1+}, \mathrm{Tl}^{3+}$ & - & $\begin{array}{l}\text { Extraction with } 1- \\
\text { pyrrolidinecarbodithioic } \\
\text { acid, APDC }\end{array}$ & Extraction-ETAAS & Wine & {$[123]$} \\
\hline $\mathrm{Tl}^{1+}, \mathrm{Tl}^{3+}$ & - & $\begin{array}{l}\text { Triton X-114, sodium } \\
\text { dodecyl sulfate, DTPA }\end{array}$ & Extraction-ICP-MS & $\begin{array}{l}\text { Environmental } \\
\text { water samples }\end{array}$ & {$[124]$} \\
\hline $\mathrm{Tl}^{1+}, \mathrm{Tl}^{3+}$ & Hamilton PRP-X100 & $\begin{array}{l}100 \mathrm{mM} \text { ammonium } \\
\text { acetate, and } 5 \mathrm{mM} \text { DTPA }\end{array}$ & IC-ICP-MS & Plants extracts & {$[125]$} \\
\hline $\mathrm{Tl}^{1+}, \mathrm{Tl}^{3+}$ & SPE (Dowex 50-8X) & $14 \% \mathrm{HNO}_{3}$ & SPE-ICP-MS & Lake waters & {$[126]$} \\
\hline $\mathrm{Tl}^{1+}, \mathrm{Tl}^{3+}$ & $\begin{array}{l}\text { Microcolumn (sodium } \\
\text { dodecyl sulfate + alumina) }\end{array}$ & $1 \mathrm{M} \mathrm{Na}_{2} \mathrm{~S}_{2} \mathrm{O}_{3}$ & FI-FAAS & $\begin{array}{l}\text { Water end } \\
\text { wastewater }\end{array}$ & {$[127]$} \\
\hline $\mathrm{Tl}^{1+}, \mathrm{Tl}^{3+}$ & - & Hydrazine & $\begin{array}{l}\text { FIA and } \\
\text { spectrofluorimetric }\end{array}$ & Real samples & {$[128]$} \\
\hline $\mathrm{Tl}^{1+}, \mathrm{Tl}^{3+}$ & Chelex-100 resin column & $3.2 \mathrm{M} \mathrm{HNO}_{3}$ & SPE-ICP-MS & Lake waters & {$[129]$} \\
\hline $\mathrm{Tl}^{1+}, \mathrm{Tl}^{3+}$ & $\begin{array}{l}\text { Microcolumn (multiwalled } \\
\text { carbon nanotubes) }\end{array}$ & $1 \mathrm{M} \mathrm{HNO}_{3}$ & SPE-STPF-ETAAS & Water & {$[130]$} \\
\hline $\mathrm{Tl}^{1+}, \mathrm{Tl}^{3+}$ & Dionex CG12A & $0.015 \mathrm{M} \mathrm{HNO}_{3}$ & $\begin{array}{l}\text { IC-ICP-OES, } \\
\text { IC-ICP-MS }\end{array}$ & Water & {$[131]$} \\
\hline $\mathrm{Tl}^{1+}, \mathrm{Tl}^{3+}$ & Dionex AG12A CG12A & $\mathrm{HNO}_{3}, \mathrm{HCl}$ & IC-ICP-MS & Water & [132] \\
\hline $\mathrm{Me}_{2} \mathrm{Tl}^{+}$ & $\begin{array}{l}\text { Microcolumn (filled with } \\
\text { AG1-X8) }\end{array}$ & $\begin{array}{l}\mathrm{NaDDTC}, \mathrm{HNO}_{3}, \\
\text { MIBK, }\end{array}$ & PTI-IDMS & Oceanic water & {$[133]$} \\
\hline
\end{tabular}

spectrophotometry, ICP-MS, laser inducted fluorescence spectrometry, or differential pulse stripping voltamperometry.

There are well-developed and described methods of antimony and arsenic analysis. On the other hand, there is a great pressure, both for the analytical chemists and toxicologists, to develop credible methodologies of thallium determination, especially in complex matrix samples. Determination of thallium and its compounds in water, snow, soil, sediments, or cement has been described in recent years [72]. Literature data concerning speciation analytics of thallium performed with various hyphenated techniques is presented in Table 3.

\section{Summary and Conclusions}

Arsenic, antimony, and thallium belong to a group of elements that, due to their physical, chemical and toxicological qualities, are particularly interesting objects of research within the framework of speciation analytics. The new approach towards the presence and role of these elements and their compounds is influenced by the constant development of analytical methods (including hyphenated techniques), toxicology, biochemistry, and environmental chemistry.

Most literature data concerns arsenic and its speciation forms whereas the least information relates to thallium and its compounds. New data connected with both the characteristics of selected speciation forms of these elements and methods enabling to determine them at lower concentration levels (in the complex matrix samples, such as food ones or living tissues) ought to emerge in the years to follow.

The hyphenated techniques, in which separation techniques are coupled with diverse selective and sensitive detection methods, are widely used in the speciation analytics of arsenic, antimony, and thallium. The hyphenated techniques create new and ever greater possibilities. Their main advantages include extremely low limits of detection and quantification, insignificant influence of interferences on the determination process, as well as very high precision and repeatability of determinations. Obviously, the hyphenated techniques pose certain limitations that involve the complexity and high price of the apparatus. As a result, they are not readily available and used in laboratories. What is more, the application of the hyphenated techniques requires excellent comprehension of analytical methodologies and the apparatus. These systems are very expensive and employed in scientific research rather than in routine analyses. Nevertheless, the hyphenated techniques have been constantly developing and gaining more and more importance, which is corroborated by the rising number of works pertaining to the subject.

\section{Abbreviations}

$\begin{array}{ll}\text { AB: } & \text { Arsenobetaine } \\ \text { AC: } & \text { Arsenecholine } \\ \text { BPHA: } & \text { N-Benzoyl-N-phenyhydroxylamine } \\ \text { DMA: } & \text { Dimethylarsinic Acid } \\ \text { EDTA: } & \text { Ethylenediaminetetraacetic acid }\end{array}$


FIA-HGAAS: $\quad$ Flow Injection Analysis coupled to Hydride Generation Atomic Absorption Spectrometry

FIA-HG-AAS: $\quad$ Flow Injection Analysis with Hydride Generation Atomic Absorption

FI-EHG-AAS: $\quad$ Flow Injection Analysis coupled to Electrochemical Hydride Generation Atomic Absorption Spectrometry

FI-HG-ICP-MS: $\quad$ Flow Injection Hydride Generation with Inductively Coupled Plasma Mass Spectrometry

HG-AFS: $\quad$ Hydride Generation coupled to Atomic Fluorescence Spectrometry HG-ICP-AES: $\quad$ Hydride Generation Inductively coupled to Plasma Atomic Emission Spectrometry

HG-SPE-ICP-MS: Solid Phase Extraction Hydride Generation Atomic Fluorescence Spectrometry

HMDE: $\quad$ Hanging Mercury Drop Electrode

HPLC- INAA: $\quad$ High Performance Liquid Chromatography with Instrumental Neutron Activation Analysis

HPLC-DF-ICP-MS: High Performance Liquid Chromatography With Double Focusing Sector Field Inductively Coupled Plasma Mass Spectrometry

HPLC-ESI-MS:

High Performance Liquid Chromatography with Electrospray Ionization Mass Spectrometry

HPLC-ETAAS: $\quad$ High Performance Liquid Chromatography with Electrothermal Atomic Absorption Spectrometry

HPLC-FAAS: $\quad$ High Performance Liquid Chromatography with Flame Atomic Absorption Spectrometry

HPLC-HGAAS: $\quad$ High Performance Liquid Chromatography coupled to Hydride Generation Atomic Absorption Spectrometry HPLC-HG-AFS: High Performance Liquid Chromatography coupled to Hydride Generation Atomic Fluorescence Spectrometry

HPLC-ICP-AES: High Performance Liquid Chromatography Inductively coupled to Plasma Atomic Emission Spectroscopy

HPLC-ICP-DRC-MS: High Performance Liquid Chromatography with Inductively Coupled to Plasma Mass Spectrometry With Dynamic Reaction Cell

HPLC-ICP-MS: High Performance Liquid Chromatography with Inductively Coupled Plasma Mass Spectrometry
HPLC- High Performance Liquid

MO-HG- Chromatography Microwave-Assisted

AAS: $\quad$ Oven Coupled to Hydride Generation Atomic Absorption Spectrometry

HPLC- High Performance Liquid

NAA: Chromatography coupled to Neutron Activation Analysis

HPLC-SF- High Performance Liquid

ICP-MS: Chromatography with Sector Field Inductively Coupled Plasma Mass Spectrometry

IC-HG- Ion Chromatography Coupled to Hydride

AFS: Generation Atomic Fluorescence Spectrometry

IC-ICP- Ion Chromatography Coupled to

MS: $\quad$ Inductively Coupled Plasma Mass Spectrometry

IC-ICP- Ion Chromatography coupled to

OES: Inductively coupled to Plasma Atomic Emission Spectroscopy

$\mathrm{Me}_{2} \mathrm{Tl}^{+}: \quad$ Dimethyl Thallium Ion

MIBK: Methyl Isobuthyl Ketone

MMA: $\quad$ Monomethylarsonic Acid

NaDDTC: Sodium Diethyldithiocarbamate

PDCA: 2,6 Dicarboxylic Acid

PTI- Positive Thermal Ionization Isotope

IDMS: Dilution Mess Spectrometry

SPE-FAAS: Solid Phase Extraction with Flame Atomic Absorption Spectrometry

SPE- $\quad$ Solid Phase Extraction Graphite Furnace

GFAAS: Atomic Absorption Spectrometry

SPE-ICP- Solid Phase Extraction With Inductively

MS: $\quad$ Coupled Plasma Mass Spectrometry

SPE-ICP- Solid Phase Extraction With Inductively

OES: $\quad$ Coupled Plasma Atomic Emission Spectroscopy

SPE-STPF- Solid Phase Extraction Stabilized

ETAAS: Temperature Platform Furnace With Electrothermal Atomic Absorption Spectrometry

TeMAs ${ }^{+}$: Tertramethylarsonium Ion

TMAH: Tetramethylammonium hydroxide

TMAI: Tetramethylarsonium Iodide

TMAO: Trimethylarsine Oxide

$\mathrm{TMSbCl}_{2}$ : Trimethylantimony Dichloride

TMSbO: Trimethylstiboxide.

\section{References}

[1] T. M. Florence, G. E. Batley, and P. Benes, "Chemical speciation in natural waters," Critical Reviews in Analytical Chemistry, vol. 3, pp. 219-296, 1980.

[2] J. A. Caruso, K. L. Sutton, and K. L. Ackley, Elemental speciation, Comprehensive Analytical Chemistry, vol. 32, Elsevier Science, 2000.

[3] A. Kot and J. Namiesńik, "The role of speciation in analytical chemistry," Trends in Analytical Chemistry, vol. 19, no. 2-3, pp. 69-79, 2000. 
[4] A. Tessier and D. R. Turner, Metal Speciation and Bioavaliability in Aquatic Systems, Wiley, New York, NY, USA, 1995.

[5] T. M. Florence and G. E. Batley, "Chemical speciation in natural waters," Critical Reviews in Analytical Chemistry, vol. 51, pp. 1-9, 1993.

[6] A. Tessier, P. G. C. Campbell, and M. Blsson, "Sequential extraction procedure for the speciation of particulate trace metals," Analytical Chemistry, vol. 51, no. 7, pp. 844-851, 1979.

[7] Ph. Queaviller, Method Performance Studies for Speciation Analysis, The Royal Society of Chemistry Cambridge, Cambridge, UK, 1997.

[8] J. R. Dean, Methods for Environmental Trace Analysis, John Wiley \& Sons, New York, NY, USA, 2003.

[9] R. Michalski, "Applications of ion chromatography for the determination of inorganic cations," Critical Reviews in Analytical Chemistry, vol. 39, no. 4, pp. 230-250, 2009.

[10] L. A. Ellis and D. J. Roberts, "Chromatographic and hyphenated methods for elemental speciation analysis in environmental media," Journal of Chromatography A, vol. 774, no. 1-2, pp. 3-19, 1997.

[11] H. Taylor, Inductively Coupled Plasma-Mass Spectrometry: Practices and Techniques, Academic Press, London, UK, 2000.

[12] A. Imran and Y. Aboul-Enein, Instrumental Methods in Metal Ion Speciation, Taylor \& Francis, 2006.

[13] M. A. Vieira, P. Grinberg, C. R. R. Bobeda, M. N. M. Reyes, and R. C. Campos, "Non-chromatographic atomic spectrometric methods in speciation analysis: a review," Spectrochimica Acta B, vol. 64, no. 6, pp. 459-476, 2009.

[14] G. F. Nordberg, B. A. Flower, M. Nordberg, and L. Friberg, Handbook of the Toxicology of Metals, Academic Press, London, UK, 3rd edition, 2007.

[15] J. Weiss, Handbook of Ion Chromatography, vol. 1-2, Wiley$\mathrm{VCH}, 2004$.

[16] R. Michalski, "Inorganic oxyhalide by-products in drinking water: ion chromatographic methods," in Encyclopedia of Chromatography, J. Cazes, Ed., vol. 2, pp. 1212-1217, 2010.

[17] T. Wang, "Liquid chromatography-inductively coupled plasma mass spectrometry (LC-ICP-MS)," Journal of Liquid Chromatography and Related Technologies, vol. 30, no. 5-7, pp. 807-831, 2007.

[18] A. Wille, S. Czyborra, and A. Steinbach, "Hyphenated techniques in ion chromatography," LC-GC Europe, vol. 20, no. 12, pp. 42-46, 2007.

[19] M. Popp, S. Hann, and G. Koellensperger, "Environmental application of elemental speciation analysis based on liquid or gas chromatography hyphenated to inductively coupled plasma mass spectrometry-a review," Analytica Chimica Acta, vol. 668, no. 2, pp. 114-129, 2010.

[20] F. Rouessac and A. Rouessac, Chemical Analysis: Modern Instrumentation Methods and Techniques, Wiley, Chichester, UK, 2000.

[21] A. K. Das, M. Guardia, and M. L. Cervera, "Literature survey of on-line elemental speciation in aqueous solutions," Talanta, vol. 55, no. 1, pp. 1-28, 2001.

[22] A. J. Bednar, J. R. Garbarino, M. R. Burkhardt, J. F. Ranville, and T. R. Wildeman, "Field and laboratory arsenic speciation methods and their application to natural-water analysis," Water Research, vol. 38, no. 2, pp. 355-364, 2004.

[23] P. Teräsahde, M. Pantsar-Kallio, and P. K. G. Manninen, "Simultaneous determination of arsenic species by ion chromatography-inductively coupled plasma mass spectrometry," Journal of Chromatography A, vol. 750, no. 1-2, pp. 83-88, 1996.
[24] L. O. Iserte, A. F. Roig-Navarro, and F. Hernández, "Simultaneous determination of arsenic and selenium species in phosphoric acid extracts of sediment samples by HPLC-ICPMS," Analytica Chimica Acta, vol. 527, no. 1, pp. 97-104, 2004.

[25] M. M. Rahman, Z. L. Chen, and R. Naidu, "Extraction of arsenic species in soils using microwave-assisted extraction detected by ion chromatography coupled to inductively coupled plasma mass spectrometry," Environmental Geochemistry and Health, vol. 31, no. 1, pp. 93-102, 2009.

[26] Z. Chen, N. I. Khan, G. Owens, and R. Naidu, "Elimination of chloride interference on arsenic speciation in ion chromatography inductively coupled mass spectrometry using an octopole collision/reaction system," Microchemical Journal, vol. 87, no. 1, pp. 87-90, 2007.

[27] R. Xie, W. Johnson, S. Spayd, G. S. Hall, and B. Buckley, "Arsenic speciation analysis of human urine using ion exchange chromatography coupled to inductively coupled plasma mass spectrometry," Analytica Chimica Acta, vol. 578, no. 2, pp. 186-194, 2006.

[28] I. B. Rodriguez, G. Raber, and W. Goessler, "Arsenic speciation in fish sauce samples determined by HPLC coupled to inductively coupled plasma mass spectrometry," Food Chemistry, vol. 112, no. 4, pp. 1084-1087, 2009.

[29] A. F. Roig-Navarro, Y. Martinez-Bravo, F. J. López, and F. Hernández, "Simultaneous determination of arsenic species and chromium(VI) by high-performance liquid chromatography-inductively coupled plasma-mass spectrometry," Journal of Chromatography A, vol. 912, no. 2, pp. 319-327, 2001.

[30] W. D. James, T. Raghvan, T. J. Gentry, G. Shan, and R. H. Loeppert, "Arsenic speciation: HPLC followed by ICP-MS or INAA," Journal of Radioanalytical and Nuclear Chemistry, vol. 278, no. 2, pp. 267-270, 2008.

[31] G. Koellensperger, J. Nurmi, S. Hann, G. Stingeder, W. J. Fitz, and W. W. Wenzel, "CE-ICP-SFMS and HPIC-ICP-SFMS for arsenic speciation in soil solution and soil water extracts," Journal of Analytical Atomic Spectrometry, vol. 17, no. 9, pp. 1042-1047, 2002.

[32] R. T. Gettar, R. N. Garavaglia, E. A. Gautier, and D. A. Batistoni, "Determination of inorganic and organic anionic arsenic species in water by ion chromatography coupled to hydride generation-inductively coupled plasma atomic emission spectrometry," Journal of Chromatography A, vol. 884, no. 1-2, pp. 211-221, 2000.

[33] B. P. Jackson and W. P. Miller, "Soluble arsenic and selenium species in fly ash/organic waste-amended soils using ion chromatography-inductively coupled plasma mass spectrometry," Environmental Science and Technology, vol. 33, no. 2, pp. 270-275, 1999

[34] M. Pantsar-Kallio and P. K. G. Manninen, "Simultaneous determination of toxic arsenic and chromium species in water samples by ion chromatography-inductively coupled plasma mass spectrometry," Journal of Chromatography A, vol. 779, no. 1-2, pp. 139-146, 1997.

[35] Y. Morita, T. Kobayashi, T. Kuroiwa, and T. Narukawa, "Study on simultaneous speciation of arsenic and antimony by HPLC-ICP-MS," Talanta, vol. 73, no. 1, pp. 81-86, 2007.

[36] S. H. Nam, H. J. Oh, H. S. Min, and J. H. Lee, "A study on the extraction and quantitation of total arsenic and arsenic species in seafood by HPLC-ICP-MS," Microchemical Journal, vol. 95, no. 1, pp. 20-24, 2010.

[37] C. Z. Liang, A. K. Farzana, R. M. Mahmudur, and N. Ravendra, "The separation of arsenic species in soils and plant 
tissues by anion-exchange chromatography with inductively coupled mass spectrometry using various mobile phases," Microchemical Journal, vol. 89, no. 1, pp. 20-28, 2008.

[38] B. Daus, J. Mattusch, R. Wennrich, and H. Weiss, "Investigation on stability and preservation of arsenic species in iron rich water samples," Talanta, vol. 58, no. 1, pp. 57-65, 2002.

[39] Y. Martínez-Bravo, A. F. Roig-Navarro, F. J. López, and F. Hernández, "Multielemental determination of arsenic, selenium and chromium(VI) species in water by high-performance liquid chromatography-inductively coupled plasma mass spectrometry," Journal of Chromatography A, vol. 926, no. 2, pp. 265-274, 2001.

[40] V. G. Mihucz, E. Tatár, I. Virág, E. Cseh, F. Fodor, and G. Záray, "Arsenic speciation in xylem sap of cucumber $(\mathrm{Cu}-$ cumis sativus L.)," Analytical and Bioanalytical Chemistry, vol. 383, no. 3, pp. 461-466, 2005.

[41] E. Sanz, R. Muñoz-Olivas, C. Cámara, M. K. Sengupta, and S. Ahamed, "Arsenic speciation in rice, straw, soil, hair and nails samples from the arsenic-affected areas of Middle and Lower Ganga plain," Journal of Environmental Science and Health A, vol. 42, no. 12, pp. 1695-1705, 2007.

[42] J. Zheng, H. Hintelmann, B. Dimock, and M. S. Dzurko, "Speciation of arsenic in water, sediment, and plants of the Moira watershed, Canada, using HPLC coupled to high resolution ICP-MS," Analytical and Bioanalytical Chemistry, vol. 377, no. 1, pp. 14-24, 2003.

[43] C. Z. Liang, A. K. Farzana, R. M. Mahmudur, and N. Ravendra, "Speciation of arsenic by ion chromatography inductively coupled plasma mass spectrometry using ammonium eluents," Journal of Separation Science, vol. 29, no. 17, pp. 2671-2676, 2006.

[44] P. Heitland and H. D. Köster, "Comparison of different medical cases in urinary arsenic speciation by fast HPLCICP-MS," International Journal of Hygiene and Environmental Health, vol. 212, no. 4, pp. 432-438, 2009.

[45] A. Raab and J. Feldmann, "Arsenic speciation in hair extracts," Analytical and Bioanalytical Chemistry, vol. 381, no. 2, pp. 332-338, 2005.

[46] V. Dufailly, T. Guérin, L. Noël, J.-M. Frémy, and D. Beauchemin, "A simple method for the speciation analysis of bio-accessible arsenic in seafood using on-line continuous leaching and ion exchange chromatography coupled to inductively coupled plasma mass spectrometry," Journal of Analytical Atomic Spectrometry, vol. 23, no. 9, pp. 1263-1268, 2008.

[47] B. P. Jackson and P. M. Bertsch, "Determination of arsenic speciation in poultry wastes by IC-ICP-MS," Environmental Science and Technology, vol. 35, no. 24, pp. 4868-4873, 2001.

[48] B. K. Mandal, Y. Ogra, K. Anzai, and K. T. Suzuki, "Speciation of arsenic in biological samples," Toxicology and Applied Pharmacology, vol. 198, no. 3, pp. 307-318, 2004.

[49] T. I. Todorov, J. W. Ejnik, F. G. Mullick, and J. A. Centeno, "Arsenic speciation in urine and blood reference materials," Microchimica Acta, vol. 151, no. 3-4, pp. 263-268, 2005.

[50] B. M. Hovanec, "Arsenic speciation in commercially available peanut butter spread by IC-ICP-MS," Journal of Analytical Atomic Spectrometry, vol. 19, no. 9, pp. 1141-1144, 2004.

[51] A. Raab, H. R. Hansen, L. Zhuang, and J. Feldmann, "Arsenic accumulation and speciation analysis in wool from sheep exposed to arsenosugars," Talanta, vol. 58, no. 1, pp. 67-76, 2002.

[52] M. van Hulle, C. Zhang, X. Zhang, and R. Cornelis, "Arsenic speciation in chinese seaweeds using HPLC-ICP-MS and HPLC-ES-MS," Analyst, vol. 127, no. 5, pp. 634-640, 2002.
[53] I. Pizarro, M. Gómez, C. Cámara, and M. A. Palacios, "Arsenic speciation in environmental and biological samples: extraction and stability studies," Analytica Chimica Acta, vol. 495, no. 1-2, pp. 85-98, 2003.

[54] S. N. Ronkart, V. Laurent, P. Carbonnelle, N. Mabon, A. Copin, and J.-P. Barthélemy, "Speciation of five arsenic species (arsenite, arsenate, MMAAV, DMAAV and AsBet) in different kind of water by HPLC-ICP-MS," Chemosphere, vol. 66, no. 4, pp. 738-745, 2007.

[55] Y. Inoue, Y. Date, K. Yoshida, H. Chen, and G. Endo, "Speciation of arsenic compounds in the urine of rats orally exposed to dimethylarsinic acid ion chromatography with ICP-MS as an element-selective detector," Applied Organometallic Chemistry, vol. 10, no. 9, pp. 707-711, 1996.

[56] S. Caroli, F. La Torre, F. Petrucci, and N. Violante, "On-line speciation of arsenical compounds in fish and mussel extracts by HPLC-ICP-MS," Environmental Science and Pollution Research, vol. 1, no. 4, pp. 205-208, 1994.

[57] U. Kohlmeyer, S. Jakubik, J. Kuballa, and E. Jantzen, "Determination of arsenic species in fish oil after acid digestion," Microchimica Acta, vol. 151, no. 3-4, pp. 249-255, 2005.

[58] S. Karthikeyan and S. Hirata, "Ion chromatography-inductively coupled plasma mass spectrometry determination of arsenic species in marine samples," Applied Organometallic Chemistry, vol. 18, no. 7, pp. 323-330, 2004.

[59] T. Guerin, M. Astruc, A. Batel, and M. Borsier, "Multielemental speciation of As, Se, Sb and Te by HPLC-ICP-MS," Talanta, vol. 44, no. 12, pp. 2201-2208, 1997.

[60] P. A. Creed, C. A. Schwegel, and J. T. Creed, "Investigation of arsenic speciation on drinking water treatment media utilizing automated sequential continuous flow extraction with IC-ICP-MS detection," Journal of Environmental Monitoring, vol. 7, no. 11, pp. 1079-1084, 2005.

[61] M. J. Kim, K. H. Ahn, and Y. Jung, "Distribution of arsenic species in mine tailings of abandoned mines from Korea," Chemosphere, vol. 49, p. 307, 2004.

[62] J. Y. Cabon and N. Cabon, "Speciation of major arsenic species in seawater by flow injection hydride generation atomic absorption spectrometry," Fresenius' Journal of Analytical Chemistry, vol. 368, no. 5, pp. 484-489, 2000.

[63] X. Yin, E. Hoffmann, and C. Lüdke, "Differential determination of arsenic (III) and total arsenic with L-cysteine as prereductant using a flow injection non-dispersive atomic absorption device," Fresenius' Journal of Analytical Chemistry, vol. 355, no. 3-4, pp. 324-326, 1996.

[64] U. Pyell, A. Dworschak, F. Nitschke, and B. Neidhart, "Flow injection electrochemical hydride generation atomic absorption spectrometry (FI-EHG-AAS) as a simple device for the speciation of inorganic arsenic and selenium," Fresenius' Journal of Analytical Chemistry, vol. 363, no. 5-6, pp. 495498, 1999.

[65] M. Chausseau, C. Roussel, N. Gilon, and J. M. Mermet, "Optimization of HPLC-ICP-AES for the determination of arsenic species," Fresenius' Journal of Analytical Chemistry, vol. 366, no. 5, pp. 476-480, 2000.

[66] Y. Shi, R. Acharya, and A. Chatt, "Speciation of arsenic in natural waters by HPLC-NAA," Journal of Radioanalytical and Nuclear Chemistry, vol. 262, no. 1, pp. 277-286, 2004.

[67] O. V. Shuvaeva, O. S. Koshcheeva, and N. F. Beisel, "Arsenic speciation in water by high-performance liquid chromatography with electrothermal atomic absorption detection," Journal of Analytical Chemistry, vol. 57, no. 11, pp. 10371041, 2002. 
[68] J. Stummeyer, B. Harazim, and T. Wippermann, "Speciation of arsenic in water samples by high-performance liquid chromatography-hydride generation-atomic absorption spectrometry at trace levels using a post-column reaction system," Fresenius' Journal of Analytical Chemistry, vol. 354, no. 3, pp. 344-351, 1996.

[69] Z. Šlejkovec, I. Salma, J. T. van Elteren, and É Zemplén-Papp, "Speciation of arsenic in coarse and fine urban aerosols using sequential extraction combined with liquid chromatography and atomic fluorescence detection," Fresenius' Journal of Analytical Chemistry, vol. 366, no. 8, pp. 830-834, 2000.

[70] Y. Chun-Gang, H. Bin, G. Er-Le, X. Jian, and J. Gui-Bin, "Evaluation of extraction methods for arsenic speciation in polluted soil and rotten ore by HPLC-HG-AFS analysis," Microchimica Acta, vol. 159, no. 1-2, pp. 175-182, 2007.

[71] M. Moldovan, M. M. Gómez, M. A. Palacios, and C. Cámara, "Arsenic speciation in water and human urine by HPLC/ICP/MS and HPLC/MO/HG/AAS," Microchemical Journal, vol. 59, no. 1, pp. 89-99, 1998.

[72] R. Cornelis, H. Crews, J. Caruso, and K. G. Heumann, Handbook of Elemental Speciation II: species in the Environment, Food, Medicine \& Occupational Health, John Wiley \& Sons, New York, NY, USA, 2005.

[73] R. A. Hites, Elements of Environmental Chemistry, John Wiley \& Sons, New York, NY, USA, 2007.

[74] N. Ulrich, P. Shaked, and D. Zilberstein, "Speciation of antimony (III) and antimony(V) in cell extracts by anion chromatography/inductively coupled plasma mass spectrometry," Fresenius' Journal of Analytical Chemistry, vol. 368, no. 1, pp. 62-66, 2000.

[75] M. Krachler and H. Emons, "Speciation analysis of antimony by high-performance liquid chromatography inductively coupled plasma mass spectrometry using ultrasonic nebulization," Analytica Chimica Acta, vol. 429, no. 1, pp. 125-133, 2001.

[76] M. Krachler and H. Emons, "Urinary antimony speciation by HPLC-ICP-MS," Journal of Analytical Atomic Spectrometry, vol. 16, no. 1, pp. 20-25, 2001.

[77] S. Amereih, T. Meisel, E. Kahr, and W. Wegscheider, "Speciation analysis of inorganic antimony in soil using HPLC-IDICP-MS," Analytical and Bioanalytical Chemistry, vol. 383, no. 7-8, pp. 1052-1059, 2005.

[78] S. Amereih, T. Meisel, R. Scholger, and W. Wegscheider, "Antimony speciation in soil samples along two Austrian motorways by HPLC-ID-ICP-MS," Journal of Environmental Monitoring, vol. 7, no. 12, pp. 1200-1206, 2005.

[79] J. Zheng, A. Iijima, and N. Furuta, "Complexation effect of antimony compounds with citric acid and its application to the speciation of antimony(III) and antimony(V) using HPLC-ICP-MS," Journal of Analytical Atomic Spectrometry, vol. 16, no. 8, pp. 812-818, 2001.

[80] J. Zheng, A. Iijima, and N. Furuta, "Complexation effect of antimony compounds with citric acid and its application to the speciation of antimony(III) and antimony(V) using HPLC-ICP-MS," Journal of Analytical Atomic Spectrometry, vol. 16, no. 8, pp. 812-818, 2001.

[81] J. Zheng, M. Ohata, and N. Furuta, "Studies on the speciation of inorganic and organic antimony compounds in airborne particulate matter by HPLC-ICP-MS," Analyst, vol. 125, no. 6, pp. 1025-1028, 2000.

[82] J. Zheng, M. Ohata, and N. Furuta, "Antimony speciation in environmental samples by using high-performance liquid chromatography coupled to inductively coupled plasma mass spectrometry," Analytical Sciences, vol. 16, no. 1, pp. 75-80, 2000.

[83] R. Miravet, E. Bonilla, J. F. López-Sánchez, and R. Rubio, "Antimony speciation in terrestrial plants. Comparative studies on extraction methods," Journal of Environmental Monitoring, vol. 7, no. 12, pp. 1207-1213, 2005.

[84] C. Yu, Q. Ca, Z. X. Guo, Z. Yang, and S. B. Khoo, "Antimony speciation by inductively coupled plasma mass spectrometry using solid phase extraction cartridges," Analyst, vol. 127, no. 10, pp. 1380-1385, 2002.

[85] K. Telford, W. Maher, F. Krikowa, and S. Foster, "Measurement of total antimony and antimony species in mine contaminated soils by ICPMS and HPLC-ICPMS," Journal of Environmental Monitoring, vol. 10, no. 1, pp. 136-140, 2008.

[86] A. Iijima, K. Sato, T. Ikeda, H. Sato, K. Kozawa, and N. Furuta, "Concentration distributions of dissolved $\mathrm{Sb}$ (III) and $\mathrm{Sb}(\mathrm{V})$ species in size-classified inhalable airborne particulate matter," Journal of Analytical Atomic Spectrometry, vol. 25, no. 3, pp. 356-363, 2010.

[87] C. Huang, B. Hu, and Z. Jiang, "Simultaneous speciation of inorganic arsenic and antimony in natural waters by dimercaptosuccinic acid modified mesoporous titanium dioxide micro-column on-line separation and inductively coupled plasma optical emission spectrometry determination," Spectrochimica Acta B, vol. 62, no. 5, pp. 454-460, 2007.

[88] J. Lintschinger, I. Koch, S. Serves, J. Feldmann, and W. R. Cullen, "Determination of antimony species with HighPerformance Liquid Chromatography using element specific detection," Fresenius' Journal of Analytical Chemistry, vol. 359, no. 6, pp. 484-491, 1997.

[89] T. Lindemann, A. Prange, W. Dannecker, and B. Neidhart, "Stability studies of arsenic, selenium, antimony and tellurium species in water, urine, fish and soil extracts using HPLC/ICP-MS," Fresenius' Journal of Analytical Chemistry, vol. 368, no. 2-3, pp. 214-220, 2000.

[90] W. Wagner, S. Sander, and G. Henze, "Trace analysis of antimony (III) and antimony (V) by adsorptive stripping voltammetry," Fresenius' Journal of Analytical Chemistry, vol. 354, no. 1, pp. 11-15, 1996.

[91] M. J. Ellwood and W. A. Maher, "Arsenic and antimony species in surface transects and depth profiles across a frontal zone: the Chatham Rise, New Zealand," Deep-Sea Research I, vol. 49, no. 11, pp. 1971-1981, 2002.

[92] S. Marcellino, H. Attar, F. Barbier, and F. Lagarde, "Heattreated Saccharomyces cerevisiae for antimony speciation and antimony(III) preconcentration in water samples," Analytica Chimica Acta, vol. 629, pp. 73-83, 2008.

[93] F. Liu, X. C. Le, A. McKnight-Whitford et al., "Antimony speciation and contamination of waters in the Xikuangshan antimony mining and smelting area, China," Environmental Geochemistry and Health, vol. 32, no. 5, pp. 401-413, 2010.

[94] H. W. Sun, F. X. Qiao, R. Suo, L. X. Li, and S. X. Liang, "Simultaneous determination of trace arsenic(III), antimony(III), total arsenic and antimony in Chinese medicinal herbs by hydride generation-double channel atomic fluorescence spectrometry," Analytica Chimica Acta, vol. 505, no. 2, pp. 255-261, 2004.

[95] E. Fuentes, H. Pinochet, I. de Gregori, and M. Potin-Gautier, "Redox speciation analysis of antimony in soil extracts by hydride generation atomic fluorescence spectrometry," Spectrochimica Acta B, vol. 58, no. 7, pp. 1279-1289, 2003.

[96] H. R. Hansen and S. A. Pergantis, "Detection of antimony species in citrus juices and drinking water stored in PET 
containers," Journal of Analytical Atomic Spectrometry, vol. 21, no. 8, pp. 731-733, 2006.

[97] I. de Gregori, W. Quiroz, H. Pinochet, F. Pannier, and M. Potin-Gautier, "Simultaneous speciation analysis of $\mathrm{Sb}(\mathrm{III}), \mathrm{Sb}(\mathrm{V})$ and $\left(\mathrm{CH}_{3}\right)_{3} \mathrm{SbCl}_{2}$ by high performance liquid chromatography-hydride generation-atomic fluorescence spectrometry detection (HPLC-HG-AFS): application to antimony speciation in sea water," Journal of Chromatography A, vol. 1091, no. 1-2, pp. 94-101, 2005.

[98] N. Miekeley, S. R. Mortari, and A. O. Schubach, "Monitoring of total antimony and its species by ICP-MS and on-line ion chromatography in biological samples from patients treated for leishmaniasis," Fresenius' Journal of Analytical Chemistry, vol. 372, no. 3, pp. 495-502, 2002.

[99] P. H. Pacheco, R. A. Gil, L. D. Martinez, G. Polla, and P. Smichowski, "A fully automated system for inorganic antimony preconcentration and speciation in urine," Analytica Chimica Acta, vol. 603, no. 1, pp. 1-7, 2007.

[100] A. Bellido-Martín, J. L. Gómez-Ariza, P. Smichowsky, and D. Sánchez-Rodas, "Speciation of antimony in airborne particulate matter using ultrasound probe fast extraction and analysis by HPLC-HG-AFS," Analytica Chimica Acta, vol. 649, no. 2, pp. 191-195, 2009.

[101] I. de Gregori, W. Quiroz, H. Pinochet, F. Pannier, and M. Potin-Gautier, "Speciation analysis of antimony in marine biota by HPLC-(UV)-HG-AFS: extraction procedures and stability of antimony species," Talanta, vol. 73, no. 3, pp. 458465, 2007.

[102] N. Ulrich, "Speciation of antimony(III), antimony(V) and trimethylstiboxide by ion chromatography with inductively coupled plasma atomic emission spectrometric and mass spectrometric detection," Analytica Chimica Acta, vol. 359, no. 3, pp. 245-253, 1998.

[103] M. Potin-Gautier, F. Pannier, W. Quiroz, H. Pinochet, and I. de Gregori, "Antimony speciation analysis in sediment reference materials using high-performance liquid chromatography coupled to hydride generation atomic fluorescence spectrometry," Analytica Chimica Acta, vol. 553, no. 1-2, pp. 214-222, 2005.

[104] P. Viñas, I. López-García, B. Merino-Meroño, and M. Hernández-Córdoba, "Liquid chromatography-hydride generation-atomic fluorescence spectrometry hybridation for antimony speciation in environmental samples," Talanta, vol. 68, no. 4, pp. 1401-1405, 2006.

[105] R. Miravet, J. F. López-Sánchez, R. Rubio, P. Smichowski, and G. Polla, "Speciation analysis of antimony in extracts of size-classified volcanic ash by HPLC-ICP-MS," Analytical and Bioanalytical Chemistry, vol. 387, no. 5, pp. 1949-1954, 2007.

[106] Y. Petit de Pen, O. Vielma, J. L. Burguera, M. Burguera, C. Rondo, and P. Carrero, "On-line determination of antimony(III) and antimony(V) in liver tissue and whole blood by flow injection-hydride generation-atomic absorption spectrometry," Talanta, vol. 55, no. 4, pp. 743-754, 2001.

[107] Z. Fan, "Speciation analysis of antimony (III) and antimony (V) by flame atomic absorption spectrometry after separation/preconcentration with cloud point extraction," Microchimica Acta, vol. 152, no. 1-2, pp. 29-33, 2005.

[108] F. Pena-Pereira, I. Lavilla, and C. Bendicho, "Headspace single-drop microextraction with in situ stibine generation for the determination of antimony (III) and total antimony by electrothermal-atomic absorption spectrometry," Microchimica Acta, vol. 164, no. 1-2, pp. 77-83, 2009.
[109] J. Lintschinger, O. Schramel, and A. Kettrup, "The analysis of antimony species by using ESI-MS and HPLC-ICP-MS," Fresenius' Journal of Analytical Chemistry, vol. 361, no. 2, pp. 96-102, 1998.

[110] T. Lindemann, A. Prange, W. Dannecker, and B. Neidhart, "Simultaneous determination of arsenic, selenium and antimony species using HPLC/ICP-MS," Fresenius' Journal of Analytical Chemistry, vol. 364, no. 5, pp. 462-466, 1999.

[111] R. Miravet, J. F. López-Sánchez, and R. Rubio, "Leachability and analytical speciation of antimony in coal fly ash," Analytica Chimica Acta, vol. 576, no. 2, pp. 200-206, 2006.

[112] H. R. Hansen and S. A. Pergantis, "Identification of Sb(V) complexes in biological and food matrixes and their stibine formation efficiency during hydride generation with ICPMS detection," Analytical Chemistry, vol. 79, no. 14, pp. 53045311, 2007.

[113] M. J. Nash, J. E. Maskall, and S. J. Hill, "Developments with anion exchange stationary phases for HPLC-ICP-MS analysis of antimony species," Analyst, vol. 131, no. 6, pp. 724-730, 2006.

[114] M. Krachler and H. Emons, "Potential of high performance liquid chromatography coupled to flow injection hydride generation atomic absorption spectrometry for the speciation of inorganic and organic antimony compounds," Journal of Analytical Atomic Spectrometry, vol. 15, no. 3, pp. 281-285, 2000.

[115] X. Zhang, R. Cornelis, and L. Mees, "Speciation of antimony(III) and antimony(V) species by using high-performance liquid chromatography coupled to hydride generation atomic absorption spectrometry," Journal of Analytical Atomic Spectrometry, vol. 13, no. 3, pp. 205-207, 1998.

[116] Y. L. Feng, H. Narasaki, H. Y. Chen, and L. C. Tian, "Speciation of antimony(III) and antimony(V) using hydride generation inductively coupled plasma atomic emission spectrometry combined with the rate of pre-reduction of antimony," Analytica Chimica Acta, vol. 386, no. 3, pp. 297304, 1999.

[117] C. B. Hymer and J. A. Caruso, "Arsenic and its speciation analysis using high-performance liquid chromatography and inductively coupled plasma mass spectrometry," Journal of Chromatography A, vol. 1045, no. 1-2, pp. 1-13, 2004.

[118] H. Zhang, Y. F. Wang, Y. Z. Shi, and Y. H. Chen, "Application of high performance liquid chromatography and hyphenated techniques in analysis of arsenic species," Spectroscopy and Spectral Analysis, vol. 27, no. 2, pp. 386-390, 2007.

[119] D. J. Butcher, "Environmental applications of arsenic speciation using atomic spectrometry detection," Applied Spectroscopy Reviews, vol. 42, no. 1, pp. 1-22, 2007.

[120] A. Imran and K.J. Chakresh, "Advances in arsenic speciation techniques," International Journal of Environmental Analytical Chemistry, vol. 84, no. 12, pp. 947-964, 2004.

[121] T. S. Lin and J. O. Nriagu, "Thallium speciation in river waters with Chelex-100 resin," Analytica Chimica Acta, vol. 395, no. 3, pp. 301-307, 1999.

[122] P. H. Pacheco, R. A. Gil, P. Smichowski, G. Polla, and L. D. Martinez, "l-Tyrosine immobilized on multiwalled carbon nanotubes: A new substrate for thallium separation and speciation using stabilized temperature platform furnaceelectrothermal atomic absorption spectrometry," Analytica Chimica Acta, vol. 656, no. 1-2, pp. 36-41, 2009.

[123] J. Cvetković, S. Arpadjan, I. Karadjova, and T. Stafilov, "Determination of thallium in wine by electrothermal atomic absorption spectrometry after extraction preconcentration," Spectrochimica Acta B, vol. 57, no. 6, pp. 1101-1106, 2002. 
[124] N. N. Meeravali and S. J. Jiang, "Ultra-trace speciation analysis of thallium in environmental water samples by inductively coupled plasma mass spectrometry after a novel sequential mixed-micelle cloud point extraction," Journal of Analytical Atomic Spectrometry, vol. 23, no. 4, pp. 555-560, 2008.

[125] B. Krasnodębska-Ostręga, M. Asztemborska, J. Golimowski, and K. Strusińska, "Determination of thallium forms in plant extracts by anion exchange chromatography with inductively coupled plasma mass spectrometry detection (IC-ICP-MS)," Journal of Analytical Atomic Spectrometry, vol. 23, no. 12, pp. 1632-1635, 2008.

[126] T. S. Lin and J. Nriagu, "Thallium speciation in the Great Lakes," Environmental Science and Technology, vol. 33, no. 19, pp. 3394-3397, 1999.

[127] S. Dadfarnia, T. Assadollahi, and A. M. Haji Shabani, "Speciation and determination of thallium by on-line microcolumn separation/preconcentration by flow injection-flame atomic absorption spectrometry using immobilized oxine as sorbent," Journal of Hazardous Materials, vol. 148, no. 1-2, pp. 446-452, 2007.

[128] A. A. Ensafi and B. Rezaei, "Speciation of thallium by flow-injection analysis with spectrofluorimetric detection," Microchemical Journal, vol. 60, no. 1, pp. 75-83, 1998.

[129] B. S. Twining, M. R. Twiss, and N. S. Fisher, "Oxidation of thallium by freshwater plankton communities," Environmental Science and Technology, vol. 37, no. 12, pp. 2720-2726, 2003.

[130] R. A. Gil, P. H. Pacheco, P. Smichowski, R. A. Olsina, and L. D. Martinez, "Speciation analysis of thallium using electrothermal AAS following on-line pre-concentration in a microcolumn filled with multiwalled carbon nanotubes," Microchimica Acta, vol. 167, no. 3-4, pp. 187-193, 2009.

[131] P. P. Coetzee, J. L. Fischer, and M. Hu, "Simultaneous separation and determination of $\mathrm{Tl}(\mathrm{I})$ and $\mathrm{Tl}(\mathrm{III})$ by IC-ICPOES and IC-ICP-MS," Water SA, vol. 29, no. 1, pp. 17-22, 2003.

[132] U. Karlsson, A. Düker, and S. Karlsson, "Separation and quantification of $\mathrm{Tl}(\mathrm{I})$ and $\mathrm{Tl}(\mathrm{III})$ in fresh water samples," Journal of Environmental Science and Health A, vol. 41, no. 7, pp. 1155-1167, 2006.

[133] O. F. Schedlbauer and K. G. Heumann, "Development of an isotope dilution mass spectrometric method for dimethylthallium speciation and first evidence of its existence in the ocean," Analytical Chemistry, vol. 71, no. 24, pp. 5459-5464, 1999.

[134] T. Gebel, "Aresnic and antimony: comparative approach on mechanistic toxicology," Chemico-Biological Interactions, vol. 107, no. 3, pp. 131-144, 1997.

[135] P. Smichowski, "Antimony in the environment as a global pollutant: a review on analytical methodologies for its determination in atmospheric aerosols," Talanta, vol. 75, no. 1, pp. 2-14, 2008.

[136] International Agency for Research on Cancer. Summary \& Evaluations, http://www.inchem.org/documents/ukpids/ukpids/ukpid38.htm.

[137] US Environmental Protection Agency, Integrated Pisk Information System (IRS) on Antymony, National Centem for Environmental Assessement, Office of Research and Developments, Washington, DC, 1999.

[138] Deutsche Forschungsgemeinschaft (DFG), Analysis of Hazardous Substances in Biological Materials, Wiley-VCH, Weinheim, Germany, 1994.
[139] M. O. Andreae, "The determination of the chemical species of some of the "hydride elements" (arsenic, antimony, tin, and germanium) in seawater: methodology and results," in Trace Metals in Seawater, C. S. Wong, E. Boyle, K. Bruland, J. D. Berton, and E. D. Goldberg, Eds., pp. 1-19, Plenum, New York, NY, USA, 1983.

[140] B. Michalke and P. Schramel, "Antimony speciation in environmental samples by interfacing capillary electrophoresis on-line to an inductively coupled plasma mass spectrometer," Journal of Chromatography A, vol. 834, no. 1-2, pp. 341-348, 1999.

[141] S. E. Manahan, Introduction to Chemistry: Fundamentals of Environmental Chemistry, CRC Press, Boca Raton, Fla, USA, 2001.

[142] F. H. Kemper and H.P. Bertman, "Thallium," in Metals and Their Compounds in the Environment: Occurrence, Analysis and Biological Relevance, E. Merian, Ed., Chapter II.29, pp. 1227-1241, Wiley-VCH, Weinheim, Germany, 1991.

[143] B. Prinz, G. H. M. Krause, and H. Stratmann, "Thallium damages in the surroundings of the Dyckerhoff cement works in Lengerich, Westphalia," Staub, Reinhaltung der Luft, vol. 39, no. 12, pp. 457-462, 1979.

[144] T. Shibamoto and M. Dekker, Chromatographic Analysis of Environmental and Food Toxicants, CRC Press, New York, NY, USA, 3rd edition, 1998. 


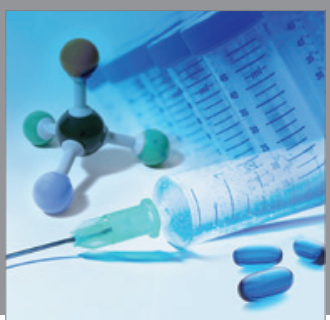

International Journal of

Medicinal Chemistry

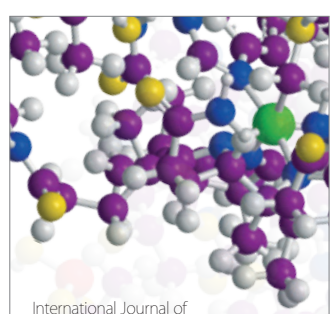

Carbohydrate Chemistry

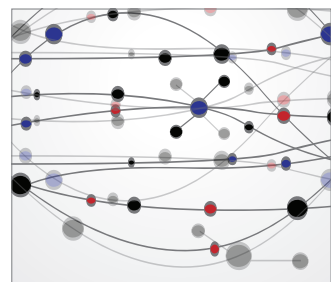

The Scientific World Journal
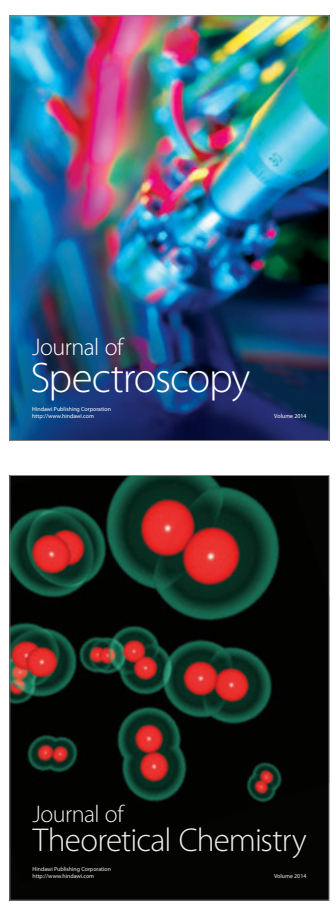
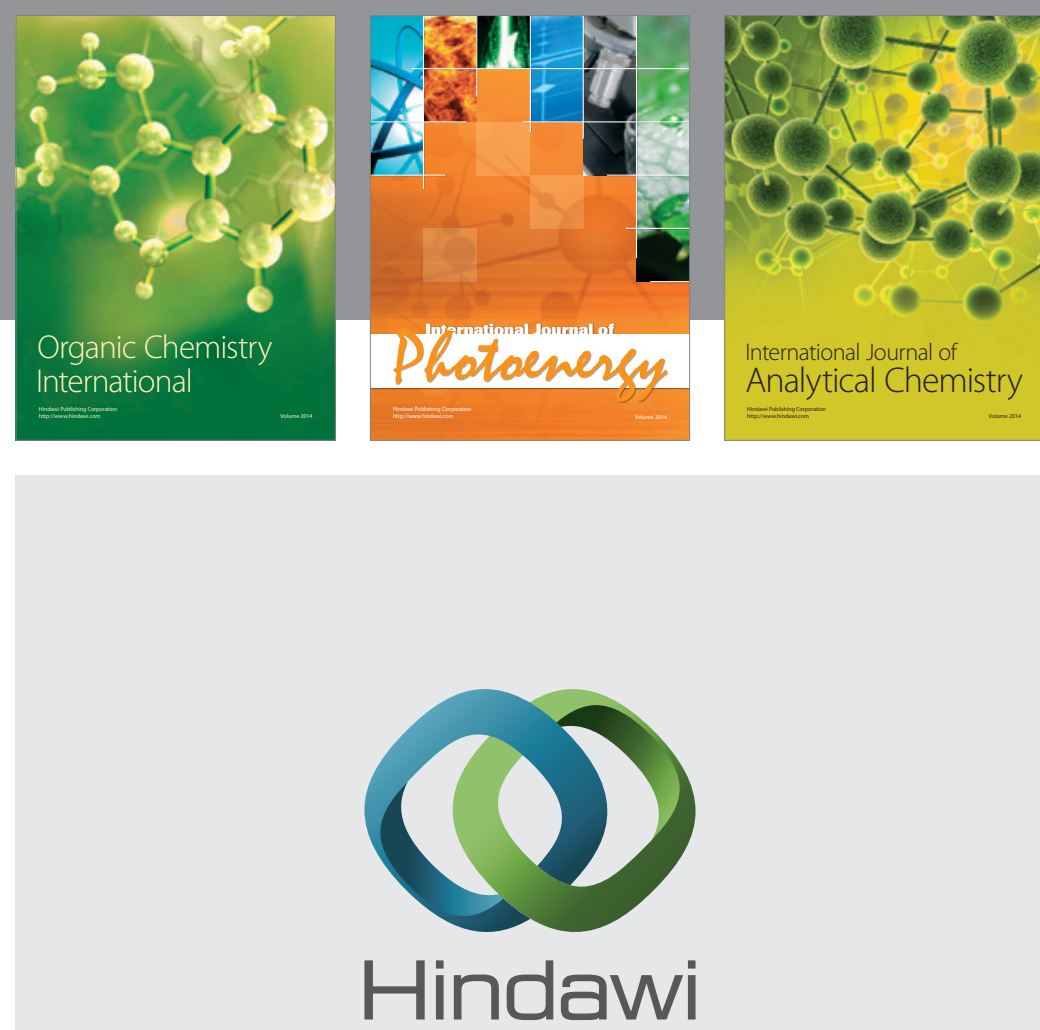

Submit your manuscripts at

http://www.hindawi.com
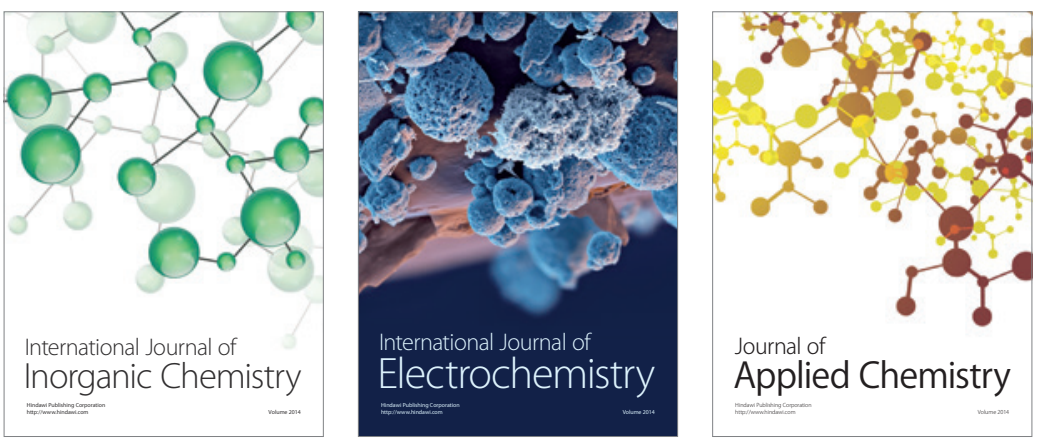

Journal of

Applied Chemistry
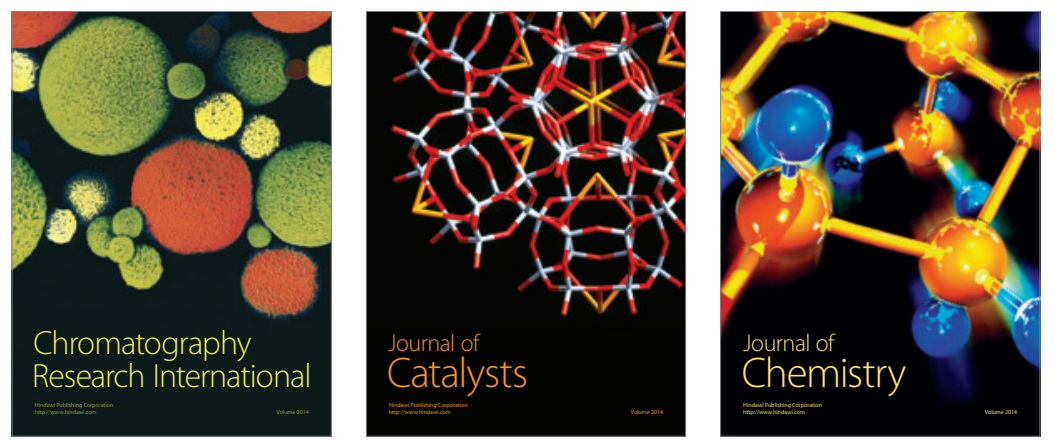
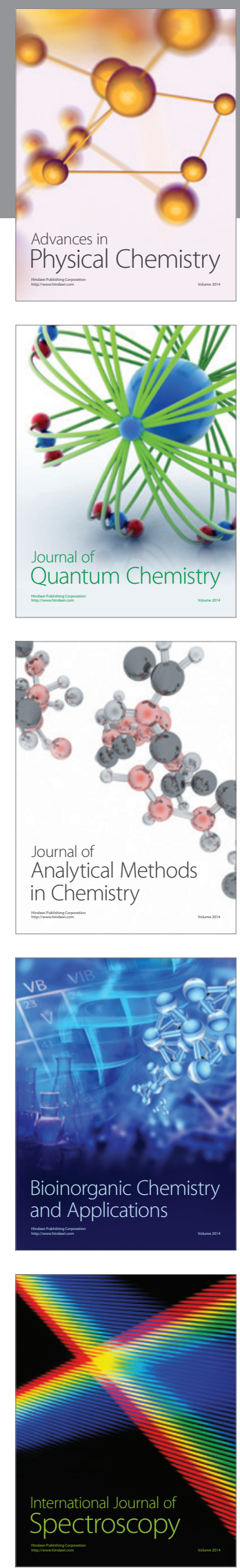